\title{
On the Eojasiewicz exponent of the gradient of a polynomial function
}

\author{
by ANDRZEJ LENARCIK (Kielce) \\ Let nothing be done through selfish ambition or conceit (Flp 2,3)
}

\begin{abstract}
Let $h=\sum h_{\alpha \beta} X^{\alpha} Y^{\beta}$ be a polynomial with complex coefficients. The Łojasiewicz exponent of the gradient of $h$ at infinity is the least upper bound of the set of all real $\lambda$ such that $|\operatorname{grad} h(x, y)| \geq c|(x, y)|^{\lambda}$ in a neighbourhood of infinity in $\mathbb{C}^{2}$, for $c>0$. We estimate this quantity in terms of the Newton diagram of $h$. Equality is obtained in the nondegenerate case.
\end{abstract}

1. Introduction. The Eojasiewicz exponent $l_{\infty}(H)$ of the polynomial mapping $H=(f, g): \mathbb{C}^{2} \rightarrow \mathbb{C}^{2}$ at infinity is the least upper bound of the set of all real $\lambda$ such that

$$
|H(z)| \geq c|z|^{\lambda}
$$

for sufficiently large $|z|$ and for $c>0$. If the set of all the exponents is empty we put $l_{\infty}(H)=-\infty$. We use the norm $|z|=\max \{|x|,|y|\}$ for $z=(x, y)$ $\in \mathbb{C}^{2}$. The quantity $l_{\infty}(H)$ is also called the exponent of growth of $H$.

Chądzyński and Krasiński [ChK1] showed that the number of solutions of the equation $f=g=0$ is finite if, and only if, $l_{\infty}(f, g)>-\infty$, and then the exponent is realized on at least one of the curves $\{f=0\}$ or $\{g=0\}$. They also proved that $l_{\infty}(H)$ is a rational number or $-\infty$. In [ChK2] they described $l_{\infty}(H)$ using the resultant. In [Pł1] Płoski gave an estimate of $l_{\infty}(H)$ for a polynomial mapping $\mathbb{C}^{n} \rightarrow \mathbb{C}^{n}$ in terms of its geometrical degree and the degrees of the mapping components. The properness of $H$ can be characterized by using $l_{\infty}(H)\left(H\right.$ is proper iff $\left.l_{\infty}(H)>0\right)$. The Eojasiewicz exponent is also applicable in the theory of polynomial automorphisms, especially the exponent of a gradient. Chạdzyński and Krasiński showed in [ChK2] that a polynomial $h: \mathbb{C}^{2} \rightarrow \mathbb{C}$ is the component of a polynomial

1991 Mathematics Subject Classification: Primary 14E05.

Key words and phrases: polynomial mapping, Łojasiewicz exponent, Newton diagram. 
automorphism if, and only if, the system of equations $\partial h / \partial X=\partial h / \partial Y=0$ has no solutions and $l_{\infty}(\operatorname{grad} h)>-1$. The connection of $l_{\infty}(\operatorname{grad} h)$ with the Newton diagram for nondegenerate $h$ was observed by P. Cassou-Noguès and Há Huy Vui [CN-H]. Some inaccuracies seem to exist in the formulation of their Proposition 10 (page 42). For $h(X, Y)=Y^{p}+X^{p}$ (page 24), $l_{\infty}(\operatorname{grad} h)=p-1$, but by the proposition this exponent equals $p$.

The aim of our paper is to give an estimate of $l_{\infty}(\operatorname{grad} h)$ in terms of the Newton diagram of $h$, without the nondegeneracy assumption. For nondegenerate polynomials, equality is obtained. Our methods are different from those in $[\mathrm{CN}-\mathrm{H}]$. The results we present in our paper are the counterparts of the results obtained by the author in the local case [L].

In the sequel we use the conventions: $\inf \emptyset=+\infty$ and $\sup \emptyset=-\infty$.

2. The main result. To present the main result we need some definitions. Let $h(X, Y)=\sum h_{\alpha \beta} X^{\alpha} Y^{\beta}$ be a polynomial with complex coefficients. We define the support of $h$ to be $\operatorname{supp} h=\left\{(\alpha, \beta): h_{\alpha \beta} \neq 0\right\}$. The degrees $\operatorname{deg} h, \operatorname{deg}_{X} h$ and $\operatorname{deg}_{Y} h$ are defined to be the maxima of $\alpha+\beta, \alpha$ and $\beta$, respectively, where $(\alpha, \beta)$ runs over $\operatorname{supp} h$. For $h=0$ we put $-\infty$ for each of the above degrees. Analogously, we define the orders $\operatorname{ord} h, \operatorname{ord}_{X} h$ and $\operatorname{ord}_{Y} h$ as the minima of the respective expressions. For $h=0$ we put $+\infty$ for each order.

The Newton diagram $\Delta_{h}$ of $h$ is the convex hull of $\operatorname{supp} h$. The set of boundary segments is the set of all one-dimensional faces which form the boundary of $\Delta_{h}$. For any boundary segment $S$ we define in $(h, S)$ as the sum of monomials $h_{\alpha \beta} x^{\alpha} y^{\beta}$ over all $(\alpha, \beta) \in S$.

We say that $h$ is nondegenerate on $S$ if the system of equations

$$
\frac{\partial}{\partial X} \operatorname{in}(h, S)=\frac{\partial}{\partial Y} \operatorname{in}(h, S)=0
$$

has no solutions in $(\mathbb{C} \backslash\{0\}) \times(\mathbb{C} \backslash\{0\})$. The right Newton polygon $\mathcal{N}_{h}^{(\mathbf{r})}$ consists of all the boundary segments which lie on the right side of $\Delta_{h}$ and join the lines $\beta=\operatorname{ord}_{Y} h$ and $\beta=\operatorname{deg}_{Y} h$. Analogously, the top Newton polygon $\mathcal{N}_{h}^{(\mathbf{t})}$ consists of all the segments which lie on the top of $\Delta_{h}$ and join the lines $\alpha=\operatorname{ord}_{X} h$ and $\alpha=\operatorname{deg}_{X} h$. The set of segments of both polygons is called the Newton polygon of $h$ at infinity. If $h$ is nondegenerate on each segment of this polygon, then we say that $h$ is nondegenerate at infinity.

Usually, the Newton polygon at infinity of a polynomial $h$ is defined to be the set of all the boundary segments, not included in the axes, for $h+$ generic const. This definition coincides with the one above if $h(X, 0) h(0, Y) \neq 0$, but differs in general (e.g. for $h=X+X^{2} Y$ ). 
We say that a segment of $\mathcal{N}_{h}^{(\mathbf{r})}$ is exceptional if it joins the horizontal axis to a point of the form $(p, 1)$. Analogously, a segment of $\mathcal{N}_{h}^{(\mathbf{t})}$ is exceptional if it joins the vertical axis to a point of the form $(1, q)$. Notice that the right polygon has no more than one exceptional segment, and similarly for the top polygon.

It is convenient to order the segments of $\mathcal{N}_{h}^{(\mathbf{r})}$ in such a way that the first segment is the nearest to the horizontal axis. Analogously in $\mathcal{N}_{h}^{(\mathbf{t})}$ the first segment is the nearest to the vertical axis. Notice that if there exists an exceptional segment of the right or top polygon, then it is the first segment of the polygon.
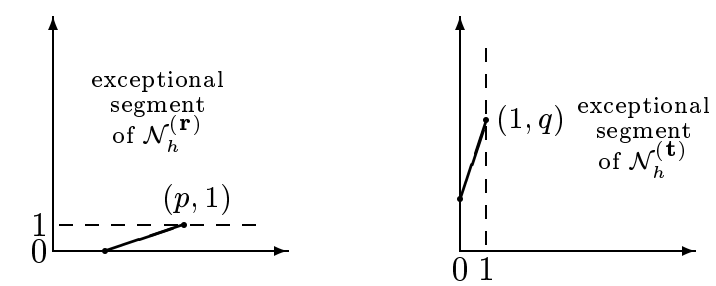

ExAmple. Let $h=X^{2}+X^{4}+X Y^{3}+X Y^{6}+X^{7} Y+X^{4} Y^{8}+X^{9} Y^{4}+$ $X^{9} Y^{6}+X^{7} Y^{8}$. The Newton polygon $\Delta_{h}$ has nine boundary segments $A, B$, $C, D, E, F, G, H, I$ which join the vertices $(2,0),(4,0),(7,1),(9,4),(9,6)$, $(7,8),(4,8),(1,6),(1,3),(2,0)$. We have $\mathcal{N}_{h}^{(\mathbf{r})}=\{B, C, D, E\}$ and $\mathcal{N}_{h}^{(\mathbf{t})}=$ $\{G, F, E\}$. The polygon of $h$ at infinity is composed of all the boundary segments except $A, H$ and $I$. Notice that $B$ is the first segment of the right polygon, $G$ is the first segment of the top polygon, and $B$ is exceptional in $\mathcal{N}_{h}^{(\mathbf{r})}$.

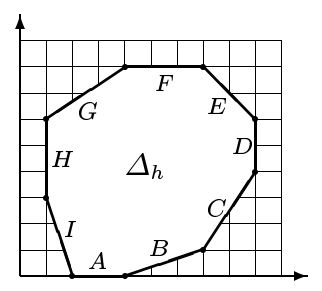

For a segment $S$ not parallel to the horizontal axis, we denote by $\alpha(S)$ the abscissa of the point where the line determined by $S$ intersects the horizontal axis. Analogously, for $S$ not parallel to the vertical axis, we denote by $\beta(S)$ the ordinate of the point where the line intersects the vertical axis. The following theorem is the main result of the paper.

TheOREM 2.1. Let $h \in \mathbb{C}[X, Y]$ be a polynomial without constant term, with nonzero gradient components, not divisible by $X^{2}$ or $Y^{2}$. If , additionally, at least one of the polygons $\mathcal{N}_{h}^{(\mathbf{r})}, \mathcal{N}_{h}^{(\mathbf{t})}$ has a segment which is not 
exceptional, then

$$
l_{\infty}(\operatorname{grad} h) \leq \min \left\{\inf _{S^{\prime}} \alpha\left(S^{\prime}\right), \inf _{S^{\prime \prime}} \beta\left(S^{\prime \prime}\right)\right\}-1,
$$

where $S^{\prime}$ runs over all the segments of the right polygon without the exceptional one, and $S^{\prime \prime}$ runs over all the segments of the top polygon without the exceptional one. Moreover, if $h$ is nondegenerate at infinity, then equality holds.

The proof of the theorem is given in Section 9. Each of the infima in the statement is a minimum if the corresponding set of segments is nonempty, and $+\infty$ otherwise. Notice that the assumption $h(0,0)=0$ is not restrictive. We can always consider $h-h(0,0)$ with the same gradient. If one of the gradient components vanishes then $l_{\infty}(\operatorname{grad} h)=0$ or $-\infty$. If $h$ is divisible by $X^{2}$ or $Y^{2}$, then, obviously, $l_{\infty}(\operatorname{grad} h)=-\infty$. The case when both the sets of segments considered are empty is described in

Proposition 2.2. Let $h \in \mathbb{C}[X, Y]$ be a polynomial without constant term, with nonzero gradient components, not divisible by $X^{2}$ or $Y^{2}$. If each of the polygons $\mathcal{N}_{h}^{(\mathbf{r})}$ and $\mathcal{N}_{h}^{(\mathbf{t})}$ is empty or has an exceptional segment only, then

$$
h(X, Y)=a X+b Y+c X Y,
$$

where $a b \neq 0$ or $c \neq 0$.

For the proof, see Propositions 9.1 and 9.2.

Under the assumptions of the proposition we have $l_{\infty}(\operatorname{grad} h)=1$ if $c \neq 0$ and $l_{\infty}(\operatorname{grad} h)=0$ if $c=0, a b \neq 0$.

The nondegeneracy of a polynomial $h$ on a boundary segment $S$ can be easily examined. If the line determined by $S$ avoids the origin, then the nondegeneracy of $h$ on $S$ is equivalent to in $(h, S)$ having no multiple factors different from $X$ and $Y$. If the line determined by $S$ passes through the origin, and $(0,0)$ is not the end of the segment, then $h$ is degenerate on $S$.

Let us return to the polynomial $h$ in the example before Theorem 2.1. It is easy to verify that all the assumptions of the theorem are satisfied. Moreover, $h$ is nondegenerate. The infima from the statement of the theorem are attained for the earliest segments, in the sense of the order in the polygons. The first segment of the right polygon, which is not exceptional, is $C$. Similarly, for the top polygon, it is $G$. Hence, $l_{\infty}(\operatorname{grad} h)=$ $\min \{\alpha(C), \beta(G)\}-1=\min \left\{6 \frac{1}{3}, 5 \frac{1}{3}\right\}-1=4 \frac{1}{3}$. The necessity of omitting the exceptional segments is overlooked in [CN-H].

As in the local case [L], the proof of Theorem 2.1 is based on the formula which describes the Łojasiewicz exponent of a pair of polynomials by using information from the Newton diagrams of both components. This is the subject of the next section. 
3. Auxiliary results. Let $H=(f, g)$ be a pair of polynomials. Our aim in this section is to describe connections between $l_{\infty}(H)$ and the Newton diagrams $\Delta_{f}$ and $\Delta_{g}$ (Theorems 3.1 and 3.2). These results are used in the proof of Theorem 2.1 in Section 9.

First, we give some definitions. We denote the projections of a segment $S$ on the horizontal and vertical axes by $S_{1}$ and $S_{2}$, respectively, and their lengths by $\left|S_{1}\right|$ and $\left|S_{2}\right|$. We now define a number $\sigma(S) \in\{-1,0,1\}$. If $S$ is parallel to one of the axes we put $\sigma(S)=0$. In other cases $\sigma(S)$ is minus the sign of the slope of $S$. In the example before Theorem 2.1 we have $\sigma=0$ for $A, D, F, H, \sigma=1$ for $E, I$, and $\sigma=-1$ for $B, C, G$.

Consider a polynomial and its global Newton polygon. The declivity of a segment $S$ of the right Newton polygon is defined to be the number $\frac{\left|S_{1}\right|}{\left|S_{2}\right|} \sigma(S)$. Obviously, it is well defined and increasing with respect to the order of the segments in the polygon. Analogously, the declivity of a segment $S$ of the top Newton polygon is $\frac{\left|S_{2}\right|}{\left|S_{1}\right|} \sigma(S)$. It is also well defined and increasing in the same sense. In the sequel, we write simply "the declivity of $S$ " if it is clear which polygon (right or top) the segment belongs to. For any nonzero $h$ and for any segment $S$ such that $\left|S_{2}\right| \neq 0$ we define

$$
\alpha\left(S, \Delta_{h}\right)=\max \left\{\alpha+\beta \frac{\left|S_{1}\right|}{\left|S_{2}\right|} \sigma(S):(\alpha, \beta) \in \operatorname{supp} h\right\},
$$

and for $S$ such that $\left|S_{1}\right| \neq 0$,

$$
\beta\left(S, \Delta_{h}\right)=\max \left\{\alpha \frac{\left|S_{2}\right|}{\left|S_{1}\right|} \sigma(S)+\beta:(\alpha, \beta) \in \operatorname{supp} h\right\} .
$$

These numbers have a simple geometrical meaning. The first is the maximal possible abscissa of the point where a line supporting $\Delta_{h}$, parallel to $S$, intersects the horizontal axis. The second is the maximal possible ordinate of the point where a line of the same type intersects the vertical axis.

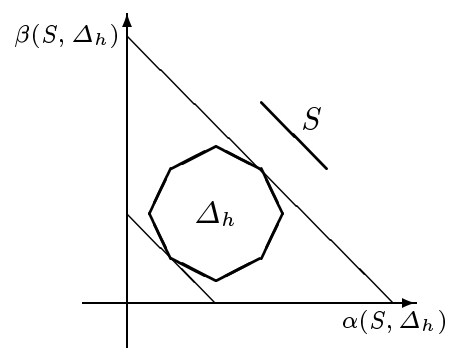

The above-introduced numbers allow us to give an upper estimate of $l_{\infty}(H)$ by using the Newton diagrams $\Delta_{f}$ and $\Delta_{g}$. We have the following 
Theorem 3.1. For a pair $H=(f, g)$ of nonzero polynomials the exponent $l_{\infty}(H)$ is bounded from above by the minimum of the following six quantities:

$$
\begin{array}{lcc}
\operatorname{deg} H(X, 0), & \inf _{S \in \mathcal{N}_{f}^{(\mathbf{r})}} \alpha\left(S, \Delta_{g}\right), & \inf _{T \in \mathcal{N}_{g}^{(\mathbf{r})}} \alpha\left(T, \Delta_{f}\right), \\
\operatorname{deg} H(0, Y), & \inf _{S \in \mathcal{N}_{f}^{(\mathbf{t})}} \beta\left(S, \Delta_{g}\right), & \inf _{T \in \mathcal{N}_{g}^{(\mathbf{t})}} \beta\left(T, \Delta_{f}\right),
\end{array}
$$

where by the degree of a pair we understand the maximum of the degrees of its components.

As earlier, we use infima to keep the quantities meaningful when the corresponding polygons are empty. The proof of the theorem is given in Section 7 . As in Theorem 2.1 we obtain equality in the nondegenerate case. First, we must give a suitable

Definition. We say that a pair $H=(f, g)$ of nonzero polynomials is nondegenerate at infinity if for any segment $S$ of the polygon of $f$ at infinity and for any segment $T$ of the polygon of $g$ at infinity one of the following conditions holds:

(a) $S$ and $T$ are not parallel.

(b) $S$ and $T$ are parallel, $S \in \mathcal{N}_{f}^{(\mathbf{r})}, T \in \mathcal{N}_{g}^{(\mathbf{r})}$ and the system of equations $\operatorname{in}(f, S)=\operatorname{in}(g, T)=0$ has no solutions in $(\mathbb{C} \backslash\{0\}) \times(\mathbb{C} \backslash\{0\})$.

(c) $S$ and $T$ are parallel, $S \in \mathcal{N}_{f}^{(\mathbf{t})}, T \in \mathcal{N}_{g}^{(\mathbf{t})}$ and the system of equations $\operatorname{in}(f, S)=\operatorname{in}(g, T)=0$ has no solutions in $(\mathbb{C} \backslash\{0\}) \times(\mathbb{C} \backslash\{0\})$.

We have the following

THEOREM 3.2. If $H=(f, g)$ is a pair of nonzero polynomials, nondegenerate at infinity, then $l_{\infty}(H)$ is equal to the minimum of the six quantities given in Theorem 3.1.

The proof is given in Section 7. Theorems 3.1 and 3.2 have a local counterpart ([L], Theorem 4.2).

ExAmple. Let $H=(f, g)$, where $f=Y^{2}+X^{4} Y^{4}+X^{5} Y^{7}+X^{3} Y^{8}$ and $g=X^{2}+X^{3}+X^{7} Y+X^{6} Y^{4}$. The polygon of $f$ at infinity consists of four segments $A, B, C, D$ which join the vertices $(0,2),(4,4),(5,7),(3,8),(0,2)$. We have $\mathcal{N}_{f}^{(\mathbf{r})}=\{A, B, C\}$ and $\mathcal{N}_{f}^{(\mathbf{t})}=\{D, C\}$. The polygon of $g$ at infinity consists of three segments $E, F, G$ which join $(3,0),(7,1),(6,4),(2,0)$. We have $\mathcal{N}_{g}^{(\mathbf{r})}=\{E, F\}$ and $\mathcal{N}_{g}^{(\mathbf{t})}=\{G, F\}$.

All the polygons considered are nonempty. In this case, the first two infima in Theorem 3.1 are attained for the segments nearest to the horizontal axis. The next two are attained for the segments nearest to the vertical axis. So, the six quantities are: $\operatorname{deg} H(X, 0)=3, \alpha\left(A, \Delta_{g}\right)=5, \alpha\left(E, \Delta_{f}\right)=-8$, 

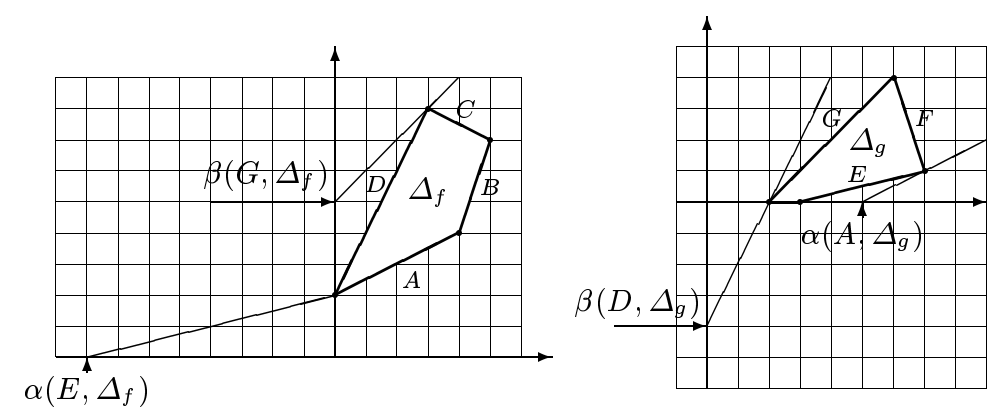

$\operatorname{deg} H(0, Y)=2, \beta\left(D, \Delta_{g}\right)=-4, \alpha\left(G, \Delta_{f}\right)=5$. Obviously, the pair is nondegenerate. From Theorem 3.2 we have $l_{\infty}(H)=\min \{3,5,-8,2,-4,5\}=-8$.

4. Relative exponents. In this section we define a few versions of the Łojasiewicz exponent relative to a variable or to a subset. They simplify the process of calculating the Łojasiewicz exponent of a polynomial mapping at infinity.

Let $H=(f, g)$ be a pair of polynomials. As in the local case ([L], [Pł2]), we can consider the relative exponent $l_{\infty}(H, X)$ which is defined to be the least upper bound of the set of all real $\lambda$ such that

$$
|H(x, y)| \geq c|x|^{\lambda}
$$

for sufficiently large $|x|$ and some $c>0$. The exponent $l_{\infty}(H, Y)$ is defined analogously. In the local situation, it is easy to verify that the Łojasiewicz exponent of a pair of series is equal to the maximum of the relative exponents. For the exponent at infinity we only have

$$
l_{\infty}(H) \geq \min \left\{l_{\infty}(H, X), l_{\infty}(H, Y)\right\} .
$$

The easy proof of equality for the local case does not carry over to the present situation if $l_{\infty}(H)<0$. However, one can verify that equality holds in (5) if $l_{\infty}(H) \geq 0$ or if $H$ is a nondegenerate pair (Corollary 7.4). An example to show that the inequality can be strict is $H=\left(1+X^{4}-Y^{2}, X^{2}-Y\right)$. By Theorem 5.4 we have $l_{\infty}(H)=-1$ and by Theorems 5.5 and $5.7, l_{\infty}(H, X)=$ -2 and $l_{\infty}(H, Y)=-1$.

A more convenient version of the relative exponent can be obtained by restriction to a subset of $\mathbb{C}^{2}$. Let $A \subset \mathbb{C}^{2}$ be an arbitrary subset. We define $l_{\infty}(H, A)$ as the upper bound of the set of all real $\lambda$ such that the inequality (1) holds for $z \in A$ and for sufficiently large $|z|$ (with some $c>0$ ). Obviously, $l_{\infty}(H, \emptyset)=+\infty$ and $l_{\infty}\left(H, \mathbb{C}^{2}\right)=l_{\infty}(H)$. One can easily verify that

$$
l_{\infty}(H, A \cup B)=\min \left\{l_{\infty}(H, A), l_{\infty}(H, B)\right\} .
$$

It is also convenient to consider an exponent relative to a variable and to a subset simultaneously. For $A \subset \mathbb{C}^{2}$ we define $l_{\infty}(H, A, X)$ as the least 
upper bound of the set of all real $\lambda$ such that (4) holds for $(x, y) \in A$ and $|x|$ sufficiently large (with some $c>0$ ). Analogously, we define $l_{\infty}(H, A, Y)$.

A starting point for calculating $l_{\infty}(H)$ is

LEMmA 4.1. Fix arbitrary constants $c_{1} \geq 1, c_{2} \geq 1$ and consider the subsets $A=\left\{|y| \leq c_{1}|x|\right\}$ and $B=\left\{|x| \leq c_{2}|y|\right\}$ of $\mathbb{C}^{2}$. Then

$$
l_{\infty}(H)=\min \left\{l_{\infty}(H, A, X), l_{\infty}(H, B, Y)\right\} .
$$

Proof. It is easy to verify that $l_{\infty}(H, A)=l_{\infty}(H, A, X)$ and $l_{\infty}(H, B)=$ $l_{\infty}(H, B, Y)$. Then we apply (6) to $A \cup B=\mathbb{C}^{2}$.

5. Laurent-Puiseux series. A convenient tool for computing the Łojasiewicz exponent at infinity (in two dimensions) is the classical technique of Laurent-Puiseux series. These are a counterpart of Newton-Puiseux series which are very useful in the local case. In this section we show how Laurent-Puiseux series can be used to calculate the exponents defined in the previous section.

Denote by $\mathbb{C}((1 / X))$ the field of formal Laurent series of the variable $X$ with the degrees of terms bounded from above. The degree of a series is the maximal power in the expansion, or $-\infty$ for the zero series. We say that $p(X)=p_{0} X^{\operatorname{deg} p}+\ldots \in \mathbb{C}((1 / X))\left(p_{0} \neq 0\right)$ is convergent if it is convergent in a neighbourhood of infinity in $\mathbb{C}$. For every such series we have

$$
c|x|^{\operatorname{deg} p} \leq|p(x)| \leq c^{\prime}|x|^{\operatorname{deg} p}
$$

for sufficiently large $|x|$, where $0<c<\left|p_{0}\right|<c^{\prime}$. Moreover, the differences $c^{\prime}-\left|p_{0}\right|$ and $\left|p_{0}\right|-c$ can be arbitrarily small. For a pair $p=\left(p_{1}, p_{2}\right)$ of series we define $\operatorname{deg} p=\max \left\{\operatorname{deg} p_{1}, \operatorname{deg} p_{2}\right\}$. An inequality of the type (7) is also valid for pairs.

The field of formal Laurent-Puiseux series (with quotient powers) is $\mathbb{C}((1 / X))^{*}=\bigcup_{k \geq 1} \mathbb{C}\left(\left(X^{-1 / k}\right)\right)$. For any nonzero Laurent-Puiseux series $a(X)=a_{0} X^{\theta_{0}}+a_{1} X^{\theta_{1}}+\ldots\left(\theta_{0}>\theta_{1}>\ldots\right)$ we put $\operatorname{deg} a(X)=\theta_{0}$ and $a^{+}(X)=a_{0} X^{\theta_{0}}$ ( $a_{0}$ will be called the leading coefficient, and $a^{+}(X)$ the leading term $)$. Moreover, $\operatorname{deg} 0=-\infty$ and $0^{+}=0$. By the definition, for any $a(X) \in \mathbb{C}((1 / X))^{*}$ there exists a positive integer $d$ such that $a\left(T^{d}\right) \in$ $\mathbb{C}((1 / T))$. We say that $a(X)$ is convergent if the corresponding $a\left(T^{d}\right)$ is convergent.

Let $h \in \mathbb{C}[X, Y]$. We say that $a(X) \in \mathbb{C}((1 / X))^{*}$ is a solution of the equation $h(X, Y)=0$ (with respect to $Y$ ) if $h(X, a(X))=0$ in $\mathbb{C}((1 / X))^{*}$. The minimal positive integer $m$ such that $\frac{\partial^{m} h}{\partial Y^{m}}(X, a(X)) \neq 0$ in $\mathbb{C}((1 / X))^{*}$ is called the multiplicity of $a(X)$ and will be denoted by $k(a)$. The polynomial $h$ can be treated as a polynomial of $Y$ with coefficients in $\mathbb{C}[X]$. If $h$ is nonzero, then the equation $h(X, Y)=0$ can be written in the form

$$
w_{0}(X) Y^{q}+w_{1}(X) Y^{q-1}+\ldots+w_{q}(X)=0, \quad w_{i} \in \mathbb{C}[X], w_{0} \neq 0,
$$


where $q=\operatorname{deg}_{Y} f$. In particular, the coefficients $w_{i}$ are elements of the field $\mathbb{C}((1 / X))^{*}$. This field is algebraically closed. This follows simply from the algebraic closedness of the analogously defined field $\mathbb{C}((X))^{*}([\mathrm{~W}]$, Chapter IV, Section 3). Hence

$$
h(X, Y)=w_{0}(X) \prod_{a \in \mathcal{H}}(Y-a(X))^{k(a)},
$$

where $\mathcal{H}$ is the set of all solutions of the above equation (we use the convention $\prod_{\emptyset}=1$ ). By passing to the local case and by using for example Artin's theorem [A], it can be derived that all the solutions are convergent. Analogously, we can solve the equation $h(X, Y)=0$ with respect to $X$ in the field $\mathbb{C}((1 / Y))^{*}$.

Further, using factorizations of the form (8), we describe the relative exponents from Lemma 4.1 by using Laurent-Puiseux series (Theorem 5.2 and 5.3). First, we prove a simple

Proposition 5.1. Let $p(T), q_{1}(T), \ldots, q_{k}(T)$ be convergent series from $\mathbb{C}((1 / T))$ such that $\operatorname{deg} p \leq d$ and $\operatorname{deg} q_{j}>d(j=1, \ldots, k)$ for a fixed positive integer $d$. Then for any $c>0$ there exists a neighbourhood $U$ of infinity in $\mathbb{C}$ such that for every $t \in U$ and $|y| \leq c|t|^{d}$,

$$
\prod_{i=1}^{k}\left|y-q_{i}(t)\right| \geq \frac{1}{2^{k}} \prod_{i=1}^{k}\left|p(t)-q_{i}(t)\right| .
$$

Proof. From the properties of the degree, for fixed $q_{i}$ there exist $r_{i}>0$ such that $\left|q_{i}(t)\right|-c|t|^{d} \geq \frac{1}{2}\left|q_{i}(t)-p(t)\right|$ for $|t|>r_{i}$. If additionally $|y| \leq c|t|^{d}$, then $\left|y-q_{i}(t)\right| \geq\left|q_{i}(t)\right|-|y| \geq\left|q_{i}(t)\right|-c|t|^{d} \geq \frac{1}{2}\left|q_{i}(t)-p(t)\right|$. Multiplying the last inequalities for $i=1, \ldots, k$, we obtain (9) with $U=\{t \in \mathbb{C}:|t|>$ $\max r_{i}$.

Now we are in a position to prove

TheOREM 5.2. Let $H=(f, g)$ be a pair of nonzero polynomials. Denote by $\mathcal{F}_{1}$ and $\mathcal{G}_{1}$, respectively, the sets of all solutions of the equations $f(X, Y)=0$ and $g(X, Y)=0$ in $\mathbb{C}((1 / X))^{*}$ with degrees not greater than 1. Then there exists a constant $c_{1} \geq 1$ such that

$$
\begin{aligned}
& l_{\infty}\left(H,\left\{|y| \leq c_{1}|x|\right\}, X\right) \\
& \quad=\min \left\{\operatorname{deg} H(X, 0), \inf _{a \in \mathcal{F}_{1}} \operatorname{deg} g(X, a(X)), \inf _{b \in \mathcal{G}_{1}} \operatorname{deg} f(X, b(X))\right\} .
\end{aligned}
$$

P r o of. We use an idea from Płoski's lemma on the norm of a polynomial mapping ([Pł2], Lemma 3.1).

Denote by $\mathcal{F}$ and $\mathcal{G}$ the sets of all solutions of $f(X, Y)=0$ and $g(X, Y)=$ 0 in $\mathbb{C}((1 / X))^{*}$, respectively. Let $d$ be a positive integer such that all the solutions have integer powers after substitution $X=T^{d}$ (we put $d=1$ if $\mathcal{F} \cup \mathcal{G}=\emptyset)$. Let $c_{1} \geq 1$ be a number greater than the moduli of the leading 
coefficients of all the solutions of degree 1 from $\mathcal{F} \cup \mathcal{G}$. Put $A=\left\{|y| \leq c_{1}|x|\right\}$. Let $p \in \mathbb{C}((1 / T))$ be a convergent series such that $\operatorname{deg} p \leq d$. If $\operatorname{deg} p=d$ then we assume that the modulus of the leading coefficient of $p$ is less than $c_{1}$. We claim that

$$
l_{\infty}(H, A, X) \leq \frac{1}{d} \operatorname{deg} H\left(T^{d}, p(T)\right) .
$$

From the properties of the degree it follows that $\left(t^{d}, p(t)\right) \in A$ for $|t|$ sufficiently large. If $H\left(T^{d}, p(T)\right)=0$ then (10) holds (both sides are $-\infty$ ). Assume that $H\left(T^{d}, p(T)\right) \neq 0$. Taking $\lambda$ as in the definition of $l_{\infty}(H, A, X)$, we have $|H(x, y)| \geq c|x|^{\lambda}$ for $(x, y) \in A$ and $|x|$ sufficiently large $(c>0)$. Then, for sufficiently large $|t|$,

$$
c^{\prime}|t|^{\operatorname{deg} H\left(T^{d}, p(T)\right)} \geq\left|H\left(t^{d}, p(t)\right)\right| \geq c\left|t^{d}\right|^{\lambda} .
$$

This means that $\operatorname{deg} H\left(T^{d}, p(T)\right) \geq \lambda d$, which gives (10). Now, the inequality $\leq$ in the theorem can be obtained by taking as $p(T)$ the zero series or any solution from $\mathcal{F}_{1} \cup \mathcal{G}_{1}$ after substitution $X=T^{d}$.

Before we prove the opposite inequality, let us make the following observation. Let $\{p(T)\}$ be a finite family, where $p(T) \in \mathbb{C}((1 / T))$ is a convergent nonzero series, or $p(T) \in \mathbb{C}((1 / T)) \times \mathbb{C}((1 / T))$ is a nonzero pair of convergent series. Notice that there exists a neighbourhood $V$ of infinity in $\mathbb{C}$ and a constant $c>0$ such that for every $t \in V$ and for every $p$ from this family,

$$
|p(t)| \geq c|t|^{\operatorname{deg} p} \text {. }
$$

Now, consider the family formed by the pair $H\left(T^{d}, 0\right)$ and all the series $f\left(T^{d}, b(T)\right)$ for $b \in \mathcal{G}_{1}$, and $g\left(T^{d}, a(T)\right)$ for $a \in \mathcal{F}_{1}$. If some element of the family is zero, then both sides of the equality in the statement of the theorem are $-\infty$. Therefore, we can assume that all the elements of the family are nonzero. Let $V$ be a neighbourhood of infinity in $\mathbb{C}$ and let $c>0$ be a constant constructed for this family as above. Let $u_{0}(X)$ be the coefficient of $Y^{\operatorname{deg}_{Y} f}$ if we treat $f$ as a polynomial of $Y$ with coefficients in $\mathbb{C}[X]$. By (8), we can write $f=f_{1} f_{2}$, where $f_{1}$ is the product of $(Y-a(X))^{k(a)}$ for $a \in \mathcal{F}_{1}(\operatorname{deg} a \leq 1)$, and $f_{2}$ is the product of such factors for $a \in \mathcal{F} \backslash \mathcal{F}_{1}$ ( $\operatorname{deg} a>1$ ) and $u_{0}(X)$. Analogously $g=g_{1} g_{2}$, where $g_{1}$ is the product of $(Y-b(X))^{k(a)}$ for $b \in \mathcal{G}_{1}(\operatorname{deg} b \leq 1)$, and $g_{2}$ is the product of such factors for $b \in \mathcal{G} \backslash \mathcal{G}_{1}(\operatorname{deg} b>1)$ and $v_{0} \in \mathbb{C}[X]$. Proposition 5.1 yields a neighbourhood $U$ of infinity in $\mathbb{C}$ such that for $t \in U$ and $|y| \leq c_{1}|t|^{d}$,

$$
\left|f_{2}\left(t^{d}, y\right)\right| \geq 2^{-\operatorname{deg}_{Y} f_{2}}\left|f_{2}\left(t^{d}, b\left(t^{d}\right)\right)\right|
$$

for every $b \in \mathcal{G}_{1} \cup\{0\}$, and

$$
\left|g_{2}\left(t^{d}, y\right)\right| \geq 2^{-\operatorname{deg}_{Y} g_{2}}\left|g_{2}\left(t^{d}, a\left(t^{d}\right)\right)\right|
$$

for every $a \in \mathcal{F}_{1} \cup\{0\}$. Now, take $(t, y)$ such that $t \in U \cap V$ and $|y| \leq c_{1}|t|^{d}$. Let $m$ denote the minimum in the statement of the theorem. If $\mathcal{F}_{1}=\mathcal{G}_{1}=\emptyset$, 
then $f=f_{2}, g=g_{2}$ and $\operatorname{deg}_{Y} f=\operatorname{deg}_{Y} f_{2}, \operatorname{deg}_{Y} g=\operatorname{deg}_{Y} g_{2}$. Applying (11) with $b=0$ and (12) with $a=0$ we have

$$
\begin{aligned}
\left|H\left(t^{d}, y\right)\right| & =\max \left\{\left|f\left(t^{d}, y\right)\right|,\left|g\left(t^{d}, y\right)\right|\right\} \\
& \geq 2^{-\max \left\{\operatorname{deg}_{Y} f, \operatorname{deg}_{Y} g\right\}} \max \left\{\left|f\left(t^{d}, 0\right)\right|, \mid g\left(t^{d}, 0\right)\right\} \\
& \geq 2^{-\operatorname{deg}_{Y} H} c\left|t^{d}\right|^{\operatorname{deg} H(X, 0)} \geq 2^{-\operatorname{deg}_{Y} H} c\left|t^{d}\right|^{m} .
\end{aligned}
$$

If $\mathcal{F}_{1}=\emptyset$ and $\mathcal{G}_{1} \neq \emptyset$, then $f=f_{2}$ and $\operatorname{deg}_{Y} f=\operatorname{deg}_{Y} f_{2}$. Let $b \in \mathcal{G}_{1}$. Using (11), we have

$$
\begin{aligned}
\left|H\left(t^{d}, y\right)\right| & \geq\left|f\left(t^{d}, y\right)\right| \geq 2^{-\operatorname{deg}_{Y} f}\left|f\left(t^{d}, b\left(t^{d}\right)\right)\right| \\
& \geq 2^{-\operatorname{deg}_{Y} f} c|t|^{\operatorname{deg} f\left(T^{d}, b\left(T^{d}\right)\right)} \geq 2^{-\operatorname{deg}_{Y} H} c\left|t^{d}\right|^{m} .
\end{aligned}
$$

The case $\mathcal{F}_{1} \neq \emptyset$ and $\mathcal{G}_{1}=\emptyset$ is analogous. If both $\mathcal{F}_{1}$ and $\mathcal{G}_{1}$ are nonempty, then we consider the finite sets $\mathcal{F}_{1}^{t}=\left\{a\left(t^{d}\right): a \in \mathcal{F}_{1}\right\}$ and $\mathcal{G}_{1}^{t}=\left\{b\left(t^{d}\right)\right.$ : $\left.b \in \mathcal{G}_{1}\right\}$ of complex numbers. Suppose that $\operatorname{dist}\left(\mathcal{F}_{1}^{t}, y\right) \geq \operatorname{dist}\left(\mathcal{G}_{1}^{t}, y\right)$. Let $\widetilde{b}\left(t^{d}\right) \in \mathcal{G}_{1}^{t}$ realize the $\operatorname{distance} \operatorname{dist}\left(y, \mathcal{G}_{1}^{t}\right)$. For every $a \in \mathcal{F}_{1}$ we have

$$
\left|y-a\left(t^{d}\right)\right| \geq \operatorname{dist}\left(y, \mathcal{F}_{1}^{t}\right) \geq \operatorname{dist}\left(y, \mathcal{G}_{1}^{t}\right)=\left|y-\widetilde{b}\left(t^{d}\right)\right|,
$$

hence $\left|y-a\left(t^{d}\right)\right| \geq \frac{1}{2}\left|y-a\left(t^{d}\right)\right|+\frac{1}{2}\left|y-\widetilde{b}\left(t^{d}\right)\right| \geq \frac{1}{2}\left|\widetilde{b}\left(t^{d}\right)-a\left(t^{d}\right)\right|$, and therefore

$$
\left|f_{1}\left(t^{d}, y\right)\right| \geq 2^{-\operatorname{deg}_{Y} f_{1}}\left|f_{1}\left(t^{d}, \widetilde{b}\left(t^{d}\right)\right)\right|,
$$

which together with (11) gives $\left|f\left(t^{d}, y\right)\right| \geq 2^{-\operatorname{deg}_{Y} f}\left|f\left(t^{d}, \widetilde{b}\left(t^{d}\right)\right)\right|$. Hence

$$
\left|H\left(t^{d}, y\right)\right| \geq\left|f\left(t^{d}, y\right)\right| \geq 2^{-\operatorname{deg}_{Y} f} c|t|^{\operatorname{deg} f\left(T^{d}, \widetilde{b}\left(T^{d}\right)\right)} \geq 2^{-\operatorname{deg} H} c\left|t^{d}\right|^{m} .
$$

The case $\operatorname{dist}\left(y, \mathcal{F}_{1}^{t}\right) \leq \operatorname{dist}\left(y, \mathcal{G}_{1}^{t}\right)$ can be verified analogously. This ends the proof of the theorem.

By symmetry we obtain

TheOREM 5.3. Let $H=(f, g)$ be a pair of nonzero polynomials. Denote by $\mathcal{F}_{1}$ and $\mathcal{G}_{1}$, respectively, the sets of all solutions of the equations $f(X, Y)=0$ and $g(X, Y)=0$ in $\mathbb{C}((1 / Y))^{*}$ with degrees not greater than 1 . Then there exists a constant $c_{2} \geq 1$ such that

$$
\begin{aligned}
l_{\infty}\left(H,\left\{|x| \leq c_{2}|y|\right\}, Y\right) & \\
& =\min \left\{\operatorname{deg} H(0, Y), \inf _{a \in \mathcal{F}_{1}} \operatorname{deg} g(a(Y), Y), \inf _{b \in \mathcal{G}_{1}} \operatorname{deg} f(b(Y), Y)\right\} .
\end{aligned}
$$

Combining Theorems 5.2 and 5.3 with Lemma 4.1 we obtain a general formula for $l_{\infty}(H)$ :

TheOREM 5.4. Let $H=(f, g)$ be a pair of nonzero polynomials. Denote by $\mathcal{F}_{1}^{\prime}$ and $\mathcal{G}_{1}^{\prime}$, respectively, the sets of all solutions of the equations $f(X, Y)=0$ and $g(X, Y)=0$ in $\mathbb{C}((1 / X))^{*}$ with degrees not greater than 1 , and by $\mathcal{F}_{1}^{\prime \prime}$ and $\mathcal{G}_{1}^{\prime \prime}$ the sets of all solutions of the same equations in $\mathbb{C}((1 / Y))^{*}$ 
with degrees also not greater than 1 . Then the exponent $l_{\infty}(H)$ is equal to the minimum of the following six quantities:

$$
\begin{array}{lcc}
\operatorname{deg} H(X, 0), & \inf _{a \in \mathcal{F}_{1}^{\prime}} \operatorname{deg} g(X, a(X)), & \inf _{b \in \mathcal{G}_{1}^{\prime}} \operatorname{deg} f(X, b(X)), \\
\operatorname{deg} H(0, Y), & \inf _{a \in \mathcal{F}_{1}^{\prime \prime}} \operatorname{deg} g(a(Y), Y), & \inf _{b \in \mathcal{G}_{1}^{\prime \prime}} \operatorname{deg} f(b(Y), Y) .
\end{array}
$$

A result of this type was obtained by Chądzyński and Krasiński in [ChK1].

The following theorem enables us to calculate $l_{\infty}(H, X)$.

Theorem 5.5. For a pair $H=(f, g)$ of nonzero polynomials,

$$
l_{\infty}(H, X)=\min \left\{\operatorname{deg} H(X, 0), \inf _{a \in \mathcal{F}} \operatorname{deg} g(X, a(X)), \inf _{b \in \mathcal{G}} \operatorname{deg} f(X, b(X))\right\},
$$

where $\mathcal{F}$ and $\mathcal{G}$ are the sets of all solutions of the equations $f(X, Y)=0$ and $g(X, Y)=0$ in $\mathbb{C}((1 / X))^{*}$, respectively.

Proof. Let $d$ be a positive integer such that all solutions have integer powers after substitution $X=T^{d}(d=1$ if $\mathcal{F} \cup \mathcal{G}=\emptyset)$. The inequality $\leq$ in the theorem can be obtained as in the proof of Theorem 5.2, by using the estimate $l_{\infty}(H, X) \leq \frac{1}{d} \operatorname{deg} H\left(T^{d}, p(T)\right)$ for $p(T) \in \mathbb{C}((1 / T))$. The opposite inequality is obtained by using an idea of Płoski [Pł2]. We employ the socalled "lemma on the norm of a polynomial mapping".

Lemma 5.6 ([Pł2], Lemma 3.1; [L], Lemma 4.7). If $w=(u, v)$ is a pair of nonzero polynomials $u(Y), v(Y) \in \mathbb{C}[Y]$, then for every $y \in \mathbb{C}$,

$$
\max \{|u(y)|,|v(y)|\} \geq 2^{-n} \min \left\{|w(0)|, \inf _{\eta \in u^{-1}(0)}|v(\eta)|, \inf _{\eta \in v^{-1}(0)}|u(\eta)|\right\},
$$

where $n=\max \{\operatorname{deg} u, \operatorname{deg} v\}$.

Now, consider the family of series $H\left(T^{d}, 0\right), f\left(T^{d}, b\left(T^{d}\right)\right)$ for $b \in \mathcal{G}$, and $g\left(T^{d}, a\left(T^{d}\right)\right.$ for $a \in \mathcal{F}$. As in the proof of Theorem 5.2 we can choose $c>0$ and a neighbourhood $V$ of infinity in $\mathbb{C}^{2}$ such that $|p(t)| \geq c|t|^{\operatorname{deg} p}$ for every $t \in V$ and for every member $p$ of the family. Now, fix $t \in V$ and $y \in \mathbb{C}$. Let $m$ be the minimum from the statement of the theorem. Using factorizations for $f$ and $g$ of the form (8), and Lemma 5.6, we obtain

$$
\begin{aligned}
\max \{\mid & \left.f\left(t^{d}, y\right)|,| g\left(t^{d}, y\right) \mid\right\} \\
& \geq 2^{-\operatorname{deg}_{Y} H} \min \left\{\left|H\left(t^{d}, 0\right)\right|, \inf _{a \in \mathcal{F}}\left|g\left(t^{d}, a\left(t^{d}\right)\right)\right|, \inf _{b \in \mathcal{G}}\left|f\left(t^{d}, b\left(t^{d}\right)\right)\right|\right\} \\
& \geq c \cdot 2^{-\operatorname{deg}_{Y} H}\left|t^{d}\right|^{m} .
\end{aligned}
$$

This ends the proof of the theorem.

By symmetry we obtain 
TheOREM 5.7. For a pair $H=(f, g)$ of nonzero polynomials,

$l_{\infty}(H, Y)=\min \left\{\operatorname{deg} H(0, Y), \inf _{a \in \mathcal{F}} \operatorname{deg} g(a(Y), Y), \inf _{b \in \mathcal{G}} \operatorname{deg} f(b(Y), Y)\right\}$,

where $\mathcal{F}$ and $\mathcal{G}$ are the sets of all solutions of the equations $f(X, Y)=0$ and $g(X, Y)=0$ in $\mathbb{C}((1 / Y))^{*}$, respectively.

6. Connections with the Newton diagram. In this section we describe connections between the degrees of the Laurent-Puiseux solutions of the equation $h(X, Y)=0$ and the Newton diagram $\Delta_{h}$ for a nonzero polynomial $h$. The results are classical. The local case is well described (see, for example, $[\mathrm{W}],[\mathrm{BK}],[\mathrm{P} t 2])$. We focus our attention on solving the equation with respect to $Y$ in $\mathbb{C}((1 / X))^{*}$. The facts concerning solutions with respect to $X$ in $\mathbb{C}((1 / Y))^{*}$ can be obtained by symmetry. We start from

Proposition 6.1. Let $h \in \mathbb{C}[X, Y]$ be a nonzero polynomial and let $S \in \mathcal{N}_{h}^{(\mathbf{r})}$. Then:

(a) There exists a factorization

$$
\operatorname{in}(h, S)=\varepsilon X^{\zeta} Y^{\vartheta} \prod_{i=1}^{\left|S_{2}\right|}\left(Y-a_{i} X^{\left(\left|S_{1}\right| /\left|S_{2}\right|\right) \sigma(S)}\right)
$$

in $\mathbb{C}((1 / X))^{*}[Y]$, where $\zeta=\sigma(S) \min \{\alpha \sigma(S):(\alpha, \beta) \in S\}, \vartheta=\min \{\beta$ : $(\alpha, \beta) \in S\}$ and $\varepsilon, a_{1}, \ldots, a_{\left|S_{2}\right|}$ are nonzero complex numbers.

(b) If $\theta$ is a rational number and $c$ is a nonzero complex number such that $\operatorname{in}(h, S)\left(X, c X^{\theta}\right)=0$, then $\theta=\frac{\left|S_{1}\right|}{\left|S_{2}\right|} \sigma(S)$ and $c$ is one of the numbers $a_{1}, \ldots, a_{\left|S_{2}\right|}$ in (a).

Proof. Notice that (b) follows immediately from (a). To prove (a) consider a factorization of in $(h, S)$ of the form

$$
\operatorname{in}(h, S)=X^{\zeta} Y^{\vartheta} \prod_{i=1}^{d}\left(u_{i} X^{\xi \sigma(S)}+v_{i} Y^{\eta}\right),
$$

where $d=\operatorname{GCD}\left\{\left|S_{1}\right|,\left|S_{2}\right|\right\}, \xi=\left|S_{1}\right| / d, \eta=\left|S_{2}\right| / d$ and $u_{i}, v_{i}$ are nonzero complex numbers. Consider a factor of the form $u X^{\xi \sigma(S)}+v Y^{\eta}$ and a new variable $X^{\prime}=X^{(\xi / \eta) \sigma(S)}=X^{\left(\left|S_{1}\right| /\left|S_{2}\right|\right) \sigma(S)}$. Then $u X^{\xi \sigma(S)}+v Y^{\eta}=u X^{\prime \eta}+$ $v Y^{\eta}=v\left(Y-\varepsilon_{1} X^{\prime}\right) \ldots\left(Y-\varepsilon_{\eta} X^{\prime}\right)$, where $\varepsilon_{1}, \ldots, \varepsilon_{\eta}$ are the complex roots of $-u / v$ of degree $\eta$. This ends the proof.

Now, fix an arbitrary rational $\theta$ and consider the linear form $(\alpha, \beta) \mapsto$ $\alpha+\theta \beta$. Since $\operatorname{supp} h$ is nonempty, the subset of supp $h$ where the form attains its maximum is also nonempty. Let $m=\max \{\alpha+\theta \beta:(\alpha, \beta) \in \operatorname{supp} h\}$. 
Consider a polynomial

$$
\sum_{\alpha+\theta \beta=m} h_{\alpha \beta} X^{\alpha} Y^{\beta}
$$

The equation $\alpha+\theta \beta=m$ describes a line supporting $\Delta_{h}$. The polynomial (13) is a monomial if the line meets the diagram in one point, or a quasi homogeneous form $\operatorname{in}(h, S)$ if the line meets the diagram along a segment $S \in \mathcal{N}_{h}^{(\mathbf{r})}$. The second possibility occurs exactly when the maximum of the linear form considered is attained at more than one point of $\operatorname{supp} h$. One can verify that this happens if and only if $\theta$ is one of the numbers $\frac{\left|S_{1}\right|}{\left|S_{2}\right|} \sigma(S)$, $S \in \mathcal{N}_{h}^{(\mathbf{r})}$. One can treat the maximum $m$ as the maximal possible degree of the substitution $h(X, a(X))$, where $a \in \mathbb{C}((1 / X))^{*}$ and $\operatorname{deg} a=\theta$. If we put $H_{\alpha \beta}=h_{\alpha \beta} X^{\alpha} a(X)^{\beta}$ for any $h_{\alpha \beta} \neq 0$, then $h(X, a(X))=\sum H_{\alpha \beta}(X)$, $H_{\alpha \beta}^{+}=h_{\alpha \beta} X^{\alpha} a^{+}(X)^{\beta}$ and $\operatorname{deg} H_{\alpha \beta}=\alpha+\theta \beta$. Using standard properties of the degree we obtain

$$
\operatorname{deg} h(X, a(X)) \leq \max \left\{\operatorname{deg} H_{\alpha \beta}\right\}=m .
$$

This observation motivates calling a substitution $h(X, a(X))$ generic if equality holds in (14), and nongeneric if strict inequality holds.

Proposition 6.2. If $h \in \mathbb{C}[X, Y]$ is a nonzero polynomial and a $(X)$ is a nonzero Laurent-Puiseux series, then the substitution $h(X, a(X))$ is nongeneric if and only if there exists a segment $S \in \mathcal{N}_{h}^{(\mathbf{r})}$ such that

$$
\operatorname{in}(h, S)\left(X, a^{+}(X)\right)=0 .
$$

Proof. We use the earlier notation. Notice that

$$
h(X, a(X))=\sum_{\alpha+\theta \beta=m} H_{\alpha \beta}^{+}+\{\text {terms of lower degree }\} .
$$

If the substitution is nongeneric, then the first term of the right hand side vanishes. This means that the number of $H_{\alpha \beta}^{+}$with maximal degree $m$ is greater than one (to obtain a reduction). In that case the sum (13) is equal to $\operatorname{in}(h, S)$ for a segment $S \in \mathcal{N}_{h}^{(\mathbf{r})}$, and then

$$
0=\sum_{\alpha+\theta \beta=m} H_{\alpha \beta}^{+}=\sum_{\alpha+\theta \beta=m} h_{\alpha \beta} X^{\alpha} a^{+}(X)^{\beta}=\operatorname{in}(h, S)\left(X, a^{+}(X)\right),
$$

which ends the proof of the "if" part.

To prove the opposite implication assume that there exists $S \in \mathcal{N}_{h}^{(\mathbf{r})}$ such that in $(h, S)\left(X, a^{+}(X)\right)=0$. By Proposition 6.1(b), $\operatorname{deg} a(X)=\frac{\left|S_{1}\right|}{\left|S_{2}\right|} \sigma(S)$ $=: \theta$. Let again $m$ be the maximum of $\alpha+\theta \beta$ over $(\alpha, \beta) \in \operatorname{supp} h$. The sum (13) is equal to $\operatorname{in}(h, S)$ in this case. The equality $\operatorname{in}\left(X, a^{+}(X)\right)=0$ means that the first term on the right side of (15) vanishes, and therefore the substitution $h(X, a(X))$ is nongeneric. 
The following theorem is a counterpart of the classical local result (for example see [Pł2], $[\mathrm{BK}])$.

Theorem 6.3. Let $h \in \mathbb{C}[X, Y]$ be a nonzero polynomial. Then:

(a) If a $(X)$ is a nonzero solution of the equation $h(X, Y)=0$ in $\mathbb{C}((1 / X))^{*}$ then there exists a segment $S \in \mathcal{N}_{h}^{(\mathbf{r})}$ such that $\operatorname{deg} a=\frac{\left|S_{1}\right|}{\left|S_{2}\right|} \sigma(S)$ and $\operatorname{in}(h, S)\left(X, a^{+}(X)\right)=0$.

(b) For every segment $S \in \mathcal{N}_{h}^{(\mathbf{r})}$ there exist exactly $\left|S_{2}\right|$ nonzero solutions in $\mathbb{C}((1 / X))^{*}$ of the equation $h(X, Y)=0$ of degree $\theta=\frac{\left|S_{1}\right|}{\left|S_{2}\right|} \sigma(S)$, counted with multiplicities. Moreover, if $\left(Y-c X^{\theta}\right)^{k}$ is a factor of $\operatorname{in}(h, S)$ in $\mathbb{C}((1 / X))^{*}[Y]$, then there exist exactly $k$ solutions, counted with multiplicities, with leading term $c X^{\theta}$.

Pr o o f. Part (a) follows immediately from Proposition 6.2. To prove (b) fix a segment $S \in \mathcal{N}_{h}^{(\mathbf{r})}$. For any nonzero $f \in \mathbb{C}((1 / X))^{*}[Y]$ we can define its weighted degree

$$
\operatorname{deg}^{*} f=\max \left\{\alpha+\beta \frac{\left|S_{1}\right|}{\left|S_{2}\right|} \sigma(S):(\alpha, \beta) \in \operatorname{supp} f\right\} .
$$

This number is well defined, because it is the maximum of a set of rationals bounded from above with bounded denominators. Define $f^{*}$ to be the sum of monomials of $f$ with maximal weighted degree. We put $\operatorname{deg}^{*} 0=-\infty$ and $0^{*}=0$. The above-defined weighted degree has standard properties: $\operatorname{deg}^{*}(f g)=\operatorname{deg}^{*} f+\operatorname{deg}^{*} g$ and $(f g)^{*}=f^{*} g^{*}$. Notice that $h^{*}=\operatorname{in}(h, S)$. Now, denote by $\mathcal{H}$ the set of all solutions of $h(X, Y)=0$ in $\mathbb{C}((1 / X))^{*}$ and consider a factorization (8) for $h$. By the properties of the weighted degree we have

$$
h^{*}=\operatorname{in}(h, S)=w_{0}(X)^{*} \prod_{a \in \mathcal{H}}\left[(Y-a(X))^{*}\right]^{k(a)},
$$

where $w_{0}(X)^{*}=w_{0}(X)^{+}$and

$$
(Y-a(X))^{*}= \begin{cases}Y & \text { if } \operatorname{deg} a(X)<\theta \\ Y-a^{+}(X) & \text { if } \operatorname{deg} a(X)=\theta \\ -a^{+}(X) & \text { if } \operatorname{deg} a(X)>\theta\end{cases}
$$

Now (b) follows immediately from the unique factorization of $\mathbb{C}((1 / X))^{*}[Y]$ and from Proposition 6.1(a).

By symmetry we can obtain results concerning solutions of $h(X, Y)=0$ in $\mathbb{C}((1 / Y))^{*}$. From Proposition 6.2 we have

Proposition 6.4. If $h \in \mathbb{C}[X, Y]$ is a nonzero polynomial and $a(Y)$ is a nonzero Laurent-Puiseux series, then the substitution $h(a(Y), Y)$ is nongeneric if and only if there exists a segment $S \in \mathcal{N}_{h}^{(\mathbf{t})}$ such that

$$
\operatorname{in}(h, S)\left(a^{+}(Y), Y\right)=0 .
$$


From Theorem 6.3 we obtain

Theorem 6.5. Let $h \in \mathbb{C}[X, Y]$ be a nonzero polynomial. Then:

(a) If $a(Y)$ is a nonzero solution of the equation $h(X, Y)=0$ in $\mathbb{C}((1 / Y))^{*}$ then there exists a segment $S \in \mathcal{N}_{h}^{(\mathbf{t})}$ such that $\operatorname{deg} a=\frac{\left|S_{2}\right|}{\left|S_{1}\right|} \sigma(S)$ and $\operatorname{in}(h, S)\left(a^{+}(Y), Y\right)=0$.

(b) For every segment $S \in \mathcal{N}_{h}^{(\mathbf{t})}$ there exist exactly $\left|S_{1}\right|$ nonzero solutions in $\mathbb{C}((1 / Y))^{*}$ of the equation $h(X, Y)=0$ of degree $\theta=\frac{\left|S_{2}\right|}{\left|S_{1}\right|} \sigma(S)$, counted with multiplicities. Moreover, if $\left(X-c Y^{\theta}\right)^{k}$ is a factor of in $(h, S)$ in $\mathbb{C}((1 / Y))^{*}[Y]$, then there exist exactly $k$ solutions, counted with multiplicities, with leading term $\mathrm{cY}^{\theta}$.

7. Proofs of auxiliary results. In this section we prove Theorems 3.1 and 3.2 by using Theorem 5.4 and the facts from the previous section. Let $h \in \mathbb{C}[X, Y]$ be a nonzero polynomial. Notice that Theorem 6.3 determines a correspondence between nonzero solutions of $h(X, Y)=0$ in $\mathbb{C}((1 / X))^{*}$ and segments of $\mathcal{N}_{h}^{(\mathbf{r})}$. To each solution $a(X)$ we can assign a segment $S \in \mathcal{N}_{h}^{(\mathbf{r})}$ such that $\operatorname{deg} a(X)=\frac{\left|S_{1}\right|}{\left|S_{2}\right|} \sigma(S)$. Analogously, Theorem 6.5 determines a similar correspondence between nonzero solutions of the equation in $\mathbb{C}((1 / Y))^{*}$ and segments of $\mathcal{N}_{h}^{(\mathbf{t})}$.

To prove Theorem 3.1 consider a pair $H=(f, g)$ of nonzero polynomials. Let $a(X) \in \mathbb{C}((1 / X))^{*}$ be a nonzero solution of $f(X, Y)=0$ and let $S \in \mathcal{N}_{f}^{(\mathbf{r})}$ correspond to this solution $\left(\operatorname{deg} a(X)=\frac{\left|S_{1}\right|}{\left|S_{2}\right|} \sigma(S)\right)$. Since the number $\alpha\left(S, \Delta_{g}\right)$, defined by (2), is exactly the maximal possible degree of the substitution $g(X, a(X))$, we have

$$
\operatorname{deg} g(X, a(X)) \leq \alpha\left(S, \Delta_{g}\right) .
$$

Now, we show that equality holds if the pair is nondegenerate. Suppose that the inequality is strict. Then the substitution is nongeneric and by Proposition 6.2 there exists $T \in \mathcal{N}_{g}^{(\mathbf{r})}$ such that $\operatorname{in}(g, T)\left(X, a^{+}(X)\right)=0$. Moreover, by Proposition 6.1(b), $\frac{\left|T_{1}\right|}{\left|T_{2}\right|} \sigma(T)=\operatorname{deg} a(X)$, so $T$ and $S$ are parallel. By Theorem 6.5(a) we also have $\operatorname{in}(f, S)\left(X, a^{+}(X)\right)=0$. Hence, the system in $(f, S)=\operatorname{in}(g, T)=0$ has a solution in $(\mathbb{C} \backslash\{0\}) \times(\mathbb{C} \backslash\{0\})$, which means that $(f, g)$ is degenerate.

Now, consider the set $\mathcal{F}_{1}^{\prime}$ of all solutions of $f(X, Y)=0$ in $\mathbb{C}((1 / X))^{*}$ of degree less than or equal to 1 . Let $\mathcal{N}_{f}^{\prime}$ be the set of $S \in \mathcal{N}_{f}^{(\mathbf{r})}$ such that $\frac{\left|S_{1}\right|}{\left|S_{2}\right|} \sigma(S) \leq 1$. For every nonzero solution in $\mathcal{F}_{1}^{\prime}$ there exists a segment of $\mathcal{N}_{f}^{\prime}$ with declivity equal to the degree of the solution. From (16) we obtain

$$
\inf _{a \in \mathcal{F}_{1}^{\prime}} \operatorname{deg} g(X, a(X)) \leq \inf _{a \in \mathcal{F}_{1}^{\prime} \backslash\{0\}} \operatorname{deg} g(X, a(X)) \leq \inf _{S \in \mathcal{N}_{f}^{\prime}} \alpha\left(S, \Delta_{g}\right) .
$$


Let $\mathcal{F}_{1}^{\prime \prime}$ be the set of all solutions of $f(X, Y)=0$ in $\mathbb{C}((1 / Y))^{*}$ of degree less than or equal to 1 . There is a correspondence between nonzero solutions of $\mathcal{F}_{1}^{\prime \prime}$ and a polygon $\mathcal{N}_{f}^{\prime \prime}$ which consists of $S \in \mathcal{N}_{f}^{(\mathbf{t})}$ with $\frac{\left|S_{2}\right|}{\left|S_{1}\right|} \sigma(S) \leq 1$. We define, analogously, the sets $\mathcal{G}_{1}^{\prime}$ and $\mathcal{G}_{1}^{\prime \prime}$ of solutions of $g(X, Y)=0$ and the polygons $\mathcal{N}_{g}^{\prime}$ and $\mathcal{N}_{g}^{\prime \prime}$. By repeating the earlier considerations for the remaining infima from the statement of Theorem 5.4, we show that $l_{\infty}(H)$ does not exceed the minimum $m$ of the six quantities

$$
\begin{array}{lcc}
\operatorname{deg} H(X, 0), & \inf _{S \in \mathcal{N}_{f}^{\prime}} \alpha\left(S, \Delta_{g}\right), & \inf _{T \in \mathcal{N}_{g}^{\prime}} \alpha\left(T, \Delta_{f}\right), \\
\operatorname{deg} H(0, Y), & \inf _{S \in \mathcal{N}_{f}^{\prime \prime}} \beta\left(S, \Delta_{g}\right), & \inf _{T \in \mathcal{N}_{g}^{\prime \prime}} \beta\left(T, \Delta_{f}\right) .
\end{array}
$$

Now, we prove equality in the case of nondegeneracy. It is sufficient to show that each of the six numbers from the statement of Theorem 5.4 is greater than or equal to $m$. For $\operatorname{deg} H(X, 0)$ and $\operatorname{deg} H(0, Y)$ this is obvious. Using equality in (16), which is already proved for nondegenerate pairs, we obtain

$$
\inf _{a \in \mathcal{F}_{1}^{\prime}} \operatorname{deg} g(X, a(X))= \begin{cases}\inf _{S \in \mathcal{N}_{f}^{\prime}} \alpha\left(S, \Delta_{g}\right) & \text { if } 0 \notin \mathcal{F}_{1}^{\prime}, \\ \min \left\{\inf _{S \in \mathcal{N}_{f}^{\prime}} \alpha\left(S, \Delta_{g}\right), \operatorname{deg} g(X, 0)\right\} & \text { if } 0 \in \mathcal{F}_{1}^{\prime} .\end{cases}
$$

In the above formula we use the fact that for each segment from $\mathcal{N}_{f}^{\prime}$ there exists a corresponding solution from $\mathcal{F}_{1}^{\prime}$ (Theorem 6.3(b)). If $0 \in \mathcal{F}_{1}^{\prime}$, then $\operatorname{deg} g(X, 0)=\operatorname{deg} H(X, 0)$. Hence, in both cases the left infimum in the formula is at least $m$. Applying the above considerations to the remaining infima from the statement of Theorem 5.4, we end the proof of the desired equality.

To complete the proof of Theorems 3.1 and 3.2 it is enough to prove

Proposition 7.1. Let $H=(f, g)$ be a pair of nonzero polynomials. Denote by $m_{1}$ the minimum of the six quantities from the statement of Theorem 3.1, and by $m$ the minimum of the six quantities in (17) and (18). Then $m_{1}=m$.

Proof. Considering inclusions of the type $\mathcal{N}^{\prime} \subset \mathcal{N}^{(\mathbf{r})}$ and $\mathcal{N}^{\prime \prime} \subset \mathcal{N}^{(\mathbf{t})}$ we have $m_{1} \leq m$. To prove the opposite inequality write

$$
\inf _{S \in \mathcal{N}_{f}^{(\mathbf{r})}} \alpha\left(S, \Delta_{g}\right)=\min \left\{\inf _{S \in \mathcal{N}_{f}^{\prime}} \alpha\left(S, \Delta_{g}\right), \inf _{S \in \mathcal{N}_{f}^{(\mathbf{r})} \backslash \mathcal{N}_{f}^{\prime}} \alpha\left(S, \Delta_{g}\right)\right\}
$$

Assume that $S \in \mathcal{N}_{f}^{(\mathbf{r})} \backslash \mathcal{N}_{f}^{\prime}$. Then $\frac{\left|S_{1}\right|}{\left|S_{2}\right|} \sigma(S)>1$, and in particular $\sigma(S)=1$. Hence

$$
\alpha+\beta \frac{\left|S_{1}\right|}{\left|S_{2}\right|} \sigma(S)=\frac{\left|S_{1}\right|}{\left|S_{2}\right|} \sigma(S)\left(\alpha \frac{\left|S_{2}\right|}{\left|S_{1}\right|} \sigma(S)+\beta\right) \geq \alpha \frac{\left|S_{2}\right|}{\left|S_{1}\right|} \sigma(S)+\beta .
$$


From (2) and (3) we obtain $\alpha\left(S, \Delta_{g}\right) \geq \beta\left(S, \Delta_{g}\right)$. By the obvious inclusion $\mathcal{N}_{f}^{(\mathbf{r})} \backslash \mathcal{N}_{f}^{\prime} \subset \mathcal{N}_{f}^{\prime \prime}$ we have

$$
\inf _{S \in \mathcal{N}_{f}^{(\mathbf{r})} \backslash \mathcal{N}_{f}^{\prime}} \alpha\left(S, \Delta_{g}\right) \geq \inf _{S \in \mathcal{N}_{f}^{(\mathbf{r})} \backslash \mathcal{N}_{f}^{\prime}} \beta\left(S, \Delta_{g}\right) \geq \inf _{S \in \mathcal{N}_{f}^{\prime \prime}} \beta\left(S, \Delta_{g}\right) .
$$

Finally,

$$
\inf _{S \in \mathcal{N}_{f}^{(\mathbf{r})}} \alpha\left(S, \Delta_{g}\right) \geq \min \left\{\inf _{S \in \mathcal{N}_{f}^{\prime}} \alpha\left(S, \Delta_{g}\right), \inf _{S \in \mathcal{N}_{f}^{\prime \prime}} \beta\left(S, \Delta_{g}\right)\right\} .
$$

Applying analogous considerations to the other three infima from the statement of Theorem 3.1 we obtain the desired inequality $m_{1} \geq m$. This ends the proof of the proposition and the proofs of Theorems 3.1 and 3.2.

From Theorem 5.5 and (16) we obtain

TheOREM 7.2. For a pair $H=(f, g)$ of nonzero polynomials,

$$
l_{\infty}(H, X) \leq \min \left\{\operatorname{deg} H(X, 0), \inf _{S \in \mathcal{N}_{f}^{(\mathbf{r})}} \alpha\left(S, \Delta_{g}\right), \inf _{T \in \mathcal{N}_{g}^{(\mathbf{r})}} \alpha\left(T, \Delta_{f}\right)\right\},
$$

with equality for a nondegenerate pair.

By symmetry, we have

Theorem 7.3. For a pair $H=(f, g)$ of nonzero polynomials,

$$
l_{\infty}(H, Y) \leq \min \left\{\operatorname{deg} H(0, Y), \inf _{S \in \mathcal{N}_{f}^{(\mathbf{t})}} \beta\left(S, \Delta_{g}\right), \inf _{T \in \mathcal{N}_{g}^{(\mathbf{t})}} \beta\left(T, \Delta_{f}\right)\right\},
$$

with equality for a nondegenerate pair.

A simple consequence of Theorem 3.2 and the above two theorems is

Corollary 7.4. If a pair $H=(f, g)$ of nonzero polynomials is nondegenerate then

$$
l_{\infty}(H)=\min \left\{l_{\infty}(H, X), l_{\infty}(H, Y)\right\} .
$$

8. Newton diagrams of derivatives. The theorems presented in the previous section give an estimate of $l_{\infty}(\operatorname{grad} h)$ for a polynomial $h(X, Y)$, based on information from the Newton diagrams of the components of the gradient. Our aim is to give an estimate of $l_{\infty}(\operatorname{grad} h)$ by using information from the Newton diagram of $h$ only. We do this by describing the structure of the right and top Newton polygons of the derivatives $\partial h / \partial X$ and $\partial h / \partial Y$. This is the main goal of this section. We focus on the right polygons. The analogous description for the top polygons can be obtained by symmetry, but will not be given explicitly.

We start with the description of the right Newton polygon of $\partial h / \partial Y$. It is easy to observe that $\operatorname{supp} \partial h / \partial Y$ is the image of $\operatorname{supp} h \backslash\{\beta=0\}$ under the translation $(\alpha, \beta) \mapsto(\alpha, \beta-1)$. We say that a segment $T$ of $\mathcal{N}_{\partial h / \partial Y}^{(\mathbf{r})}$ is 
standard if it is the image of a segment $S$ of $\mathcal{N}_{h}^{(\mathbf{r})}$ under this translation. We then write $T=S-(0,1)$. We have

$$
\operatorname{in}\left(\frac{\partial h}{\partial Y}, T\right)=\frac{\partial}{\partial Y} \operatorname{in}(h, S)
$$

in this case. Obviously nonstandard segments can also exist. Now, we are going to describe the structure of standard and nonstandard segments of the polygon considered. We order the right vertices of $\Delta_{h}$ according to their ordinates. The first one is the nearest to the horizontal axis.

Note the following simple facts. The right Newton polygon of a polynomial is nonempty if and only if its support contains at least two points with different ordinates. Hence if the right polygon of $\partial h / \partial Y$ is nonempty then the right polygon of $h$ is also nonempty. So, a necessary condition for $\mathcal{N}_{\partial h / \partial Y}^{(\mathbf{r})}$ to be nonempty is $\mathcal{N}_{h}^{(\mathbf{r})} \neq \emptyset$. The image of any right vertex of $\Delta_{h}$ with positive ordinate under the translation $(\alpha, \beta) \mapsto(\alpha, \beta-1)$ is a right vertex of $\Delta_{\partial h / \partial Y}$. Similarly, if a segment $S \in \mathcal{N}_{h}^{(\mathbf{r})}$ does not touch the horizontal axis, then $T=S-(0,1)$ is a standard segment of $\mathcal{N}_{\partial h / \partial Y}^{(\mathbf{r})}$. In particular, if the first right vertex of $\Delta_{h}$ does not lie on the horizontal axis, then all the segments of $\mathcal{N}_{\partial h / \partial Y}^{(\mathbf{r})}$ are standard. So, a necessary condition for $\mathcal{N}_{\partial h / \partial Y}^{(\mathbf{r})}$ to have nonstandard segments is: the first right vertex of $\Delta_{h}$ has to lie on the horizontal axis.

Consider a right vertex $(\mu, \nu)$ of $\Delta_{h}$ with minimal positive ordinate. From the above considerations it follows that each segment of $\mathcal{N}_{\partial h / \partial Y}^{(\mathbf{r})}$ over the vertex $(\mu, \nu-1)$ is standard, and each segment below it is nonstandard. So, a nonstandard segment of $\mathcal{N}_{\partial h / \partial Y}^{(\mathbf{r})}$ can only exist in the strip $\{0 \leq \beta \leq$ $\nu-1\}$. Now, we give a sufficient condition for $\mathcal{N}_{\partial h / \partial Y}^{(\mathbf{r})}$ to have nonstandard segments.

Proposition 8.1. Suppose that the polygon $\mathcal{N}_{h}^{(\mathbf{r})}$ is nonempty and the first right vertex of $\Delta_{h}$ lies on the horizontal axis. Then this vertex is the lower end of the first segment $F$ of the polygon, and the upper end $(\mu, \nu)$ of $F$ is the right vertex of $\Delta_{h}$ with minimal positive ordinate. Under the above assumptions, the polygon $\mathcal{N}_{\partial h / \partial Y}^{(\mathbf{r})}$ has at least one nonstandard segment if and only if there exists $(\alpha, \beta) \in \operatorname{supp} h$ such that $0<\beta<\nu$. Moreover:

(a) For every nonstandard segment $T \in \mathcal{N}_{\partial h / \partial Y}^{(\mathbf{r})}$,

$$
\frac{\left|T_{1}\right|}{\left|T_{2}\right|} \sigma(T) \leq \frac{\left|F_{1}\right|}{\left|F_{2}\right|} \sigma(F)
$$


(b) If $T \in \mathcal{N}_{\partial h / \partial Y}^{(\mathbf{r})}$ is a nonstandard segment parallel to $F$ then

$$
\operatorname{in}\left(\frac{\partial h}{\partial Y}, T\right)=\frac{\partial}{\partial Y} \operatorname{in}(h, F) .
$$

Before the proof let us consider the following

EXAMPLE. Let $h=X^{2} Y^{2}+X^{7}+X Y^{6}+X^{8} Y^{3}+X^{3} Y^{9}+X^{9} Y^{6}+X^{6} Y^{9}$.
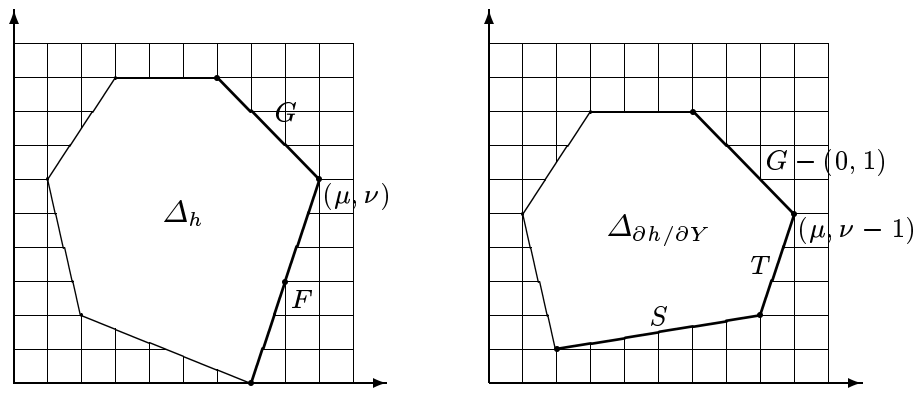

The right polygon of $h$ consists of two segments $F, G$ which join the vertices $(7,0),(9,6)$ and $(6,9)$. The first right vertex of $\Delta_{h}$ with minimal positive ordinate is $(\mu, \nu)=(9,6)$. There are two points of supp $h$ which satisfy the condition $\{0<\beta<\nu\}$. We have $\partial h / \partial Y=2 X^{2} Y+6 X Y^{5}+$ $3 X^{8} Y^{2}+9 X^{3} Y^{8}+6 X^{9} Y^{5}+9 X^{6} Y^{8}$. The right polygon of $\partial h / \partial Y$ consists of two nonstandard segments $S, T$ and one standard $G-(0,1)$. The standard and nonstandard segments are separated by the vertex $(\mu, \nu-1)=(9,5)$. The declivities of the nonstandard segments do not exceed the declivity of $F$. The segment $T$ is parallel to $F$ and obviously satisfies the condition (b).

Now, let us modify the polynomial $h$ by omitting the points $(2,2)$ and $(8,3)$ from the support. We obtain $h=X^{7}+X Y^{6}+X^{3} Y^{9}+X^{9} Y^{6}+X^{6} Y^{9}$.
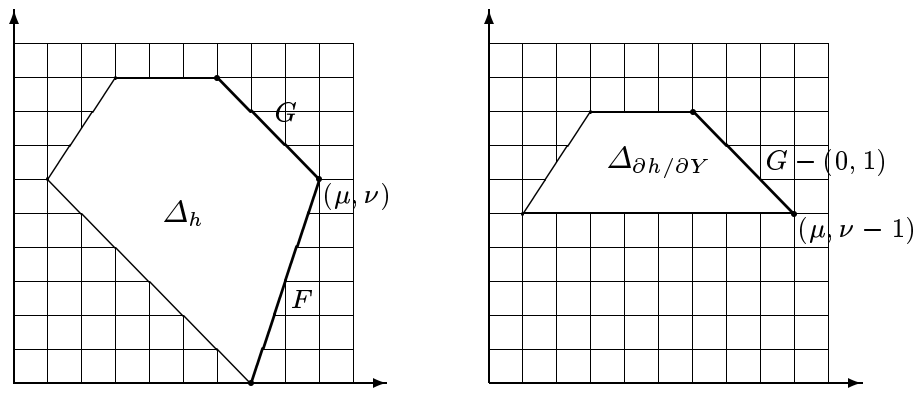

The right polygon of $h$ is the same as before, but the right polygon of $\partial h / \partial Y=6 X Y^{5}+9 X^{3} Y^{8}+6 X^{9} Y^{5}+9 X^{6} Y^{8}$ has only one standard segment $G-(0,1)$. The reason for the lack of nonstandard segments is the lack of points of $\operatorname{supp} h$ which satisfy $\{0<\beta<\nu\}$. 
Proof of Proposition 8.1. Let $T$ be a nonstandard segment of $\mathcal{N}_{\partial h / \partial Y}^{(\mathbf{r})}$. It lies in the strip $\{0 \leq \beta \leq \nu-1\}$, hence the lower end of $T+(0,1)$ belongs to supp $h$ and satisfies $0<\beta<\nu$. To prove the opposite implication, assume that $\operatorname{supp} h \cap\{0<\beta<\nu\}$ is nonempty. Then so is $\operatorname{supp} \partial h / \partial Y \cap\{\beta<\nu-1\}$. This means that the ordinate of the right vertex $(\mu, \nu-1)$ of $\Delta_{\partial h / \partial Y}$ is not minimal in supp $\partial h / \partial Y$. Hence, there exists $T \in \mathcal{N}_{\partial h / \partial Y}^{(\mathbf{r})}$ with upper end $(\mu, \nu-1)$, and clearly it is nonstandard. This ends the proof of the implication.

To prove (a) consider a nonstandard segment $T$ of $\mathcal{N}_{\partial h / \partial Y}^{(\mathbf{r})}$. We can assume that $T$ has maximal declivity. This means that $(\mu, \nu-1)$ is its upper end. Notice that the lower end of $F$ maximizes the function $(\alpha, \beta) \mapsto \frac{\alpha-\mu}{\nu-\beta}$ in $\operatorname{supp} h \cap\{\beta<\nu\}$, and the maximum is equal to the declivity of $F$. Let $\left(\alpha_{0}, \beta_{0}\right)$ be the lower end of $T$. Since $\left(\alpha_{0}, \beta_{0}+1\right) \in \operatorname{supp} h$, we have

$$
\frac{\left|T_{1}\right|}{\left|T_{2}\right|} \sigma(T)=\frac{\alpha_{0}-\mu}{(\nu-1)-\beta_{0}}=\frac{\alpha-\mu}{\nu-\left(\beta_{0}+1\right)} \leq \frac{\left|F_{1}\right|}{\left|F_{2}\right|} \sigma(F) .
$$

The segment $T$ has maximal declivity in the set of all nonstandard segments of $\mathcal{N}_{h}^{(\mathbf{r})}$. So, (a) is proved.

To prove (b) notice that if $T \in \mathcal{N}_{\partial h / \partial Y}^{(\mathbf{r})}$ is a nonstandard segment parallel to $F$, then it has maximal declivity and then $(\mu, \nu-1)$ is its upper end. In this case $T+(0,1) \subset F$ and

$$
\operatorname{in}(h, F)=\sum_{(\alpha, \beta) \in T+(0,1)} h_{\alpha \beta} X^{\alpha} Y^{\beta}+h_{p, 0} X^{p},
$$

where $(p, 0)$ is the lower end of $F$. We end the proof by differentiating the formula with respect to $Y$.

A straightforward consequence of Proposition 8.1 is

Corollary 8.2. Let $h(X, Y) \in \mathbb{C}[X, Y]$ and let $T \in \mathcal{N}_{\partial h / \partial Y}^{(\mathbf{r})}$. Then:

(a) If $T$ is parallel to a segment $S \in \mathcal{N}_{h}^{(\mathbf{r})}$, then

$$
\text { in }\left(\frac{\partial h}{\partial Y}, T\right)=\frac{\partial}{\partial Y} \operatorname{in}(h, S) \text {. }
$$

(b) If $T$ is not parallel to any segment from $\mathcal{N}_{h}^{(\mathbf{r})}$, then the declivity of $T$ is less than all the declivities of segments $S \in \mathcal{N}_{h}^{(\mathbf{r})}$; such a $T$ can only exist if the first right vertex of $\Delta_{h}$ lies on the horizontal axis.

Now, we describe the structure of the right polygon of $\partial h / \partial X$. Notice that $\operatorname{supp} \partial h / \partial X$ is the $(-1,0)$-translate of $\operatorname{supp} h \backslash\{\alpha=0\}$. We say that a segment $R \in \mathcal{N}_{\partial h / \partial X}^{(\mathbf{r})}$ is standard if it is the $(-1,0)$-translate of a segment 
$S \in \mathcal{N}_{h}^{(\mathbf{r})}$. We write $R=S-(1,0)$. Obviously

$$
\operatorname{in}\left(\frac{\partial h}{\partial X}, R\right)=\frac{\partial}{\partial X} \operatorname{in}(h, S)
$$

in this case. As earlier, a necessary condition for $\mathcal{N}_{\partial h / \partial X}^{(\mathbf{r})}$ to be nonempty is $\mathcal{N}_{h}^{(\mathbf{r})} \neq \emptyset$. The $(-1,0)$-translate of any right vertex of $\Delta_{h}$ with positive abscissa is a right vertex of $\Delta_{\partial h / \partial X}$. Similarly, if $S \in \mathcal{N}_{h}^{(\mathbf{r})}$ does not touch the vertical axis, then $R=S-(1,0)$ is a standard segment of $\mathcal{N}_{\partial h / \partial X}^{(\mathbf{r})}$. Assume that $\partial h / \partial X \neq 0$. Let $\left(\mu_{1}, \nu_{1}\right)$ be the first right vertex of $\Delta_{h}$ with positive abscissa and let $\left(\mu_{2}, \nu_{2}\right)$ be the last one with this property. We have $\nu_{1} \leq \nu_{2}$. Clearly, every segment $R \in \mathcal{N}_{\partial h / \partial X}^{(\mathbf{r})}$ in the strip $\left\{\nu_{1} \leq \beta \leq \nu_{2}\right\}$ is standard and every segment in one of the two strips $\left\{0 \leq \beta \leq \nu_{1}\right\},\left\{\nu_{2} \leq \beta \leq \operatorname{deg}_{Y} h\right\}$ is nonstandard. So, we can introduce, in a natural way, lower and upper nonstandard segments of the polygon considered. A necessary condition for the existence of lower nonstandard segments of $\mathcal{N}_{\partial h / \partial X}^{(\mathbf{r})}$ is that the first right vertex of $\Delta_{h}$ has to lie on the vertical axis. The analogous necessary condition for upper nonstandard segments is that the last right vertex of $\Delta_{h}$ has to lie on the vertical axis.

In the following two propositions we give sufficient conditions for the existence of nonstandard segments of both types. The proofs are analogous to the proof of Proposition 8.1.

Proposition 8.3. Suppose that $\mathcal{N}_{h}^{(\mathbf{r})}$ is nonempty and the first right vertex of $\Delta_{h}$ lies on the vertical axis. Let $F$ be the first segment of $\mathcal{N}_{h}^{(\mathbf{r})}$. Then the polygon $\mathcal{N}_{\partial h / \partial X}^{(\mathbf{r})}$ has at least one lower nonstandard segment if and only if there exists a point of $\operatorname{supp} h$ with positive abscissa in the interior of the strip $\mathbb{R} \times F_{2}\left({ }^{1}\right)$. Moreover:

(a) For every lower nonstandard segment $R \in \mathcal{N}_{\partial h / \partial X}^{(\mathbf{r})}$ we have

$$
\frac{\left|R_{1}\right|}{\left|R_{2}\right|} \sigma(R) \leq \frac{\left|F_{1}\right|}{\left|F_{2}\right|} \sigma(F) .
$$

(b) If $R \in \mathcal{N}_{\partial h / \partial X}^{(\mathbf{r})}$ is a lower nonstandard segment parallel to $F$, then

$$
\operatorname{in}\left(\frac{\partial h}{\partial X}, R\right)=\frac{\partial}{\partial X} \operatorname{in}(h, F) .
$$

Proposition 8.4. Suppose that $\mathcal{N}_{h}^{(\mathbf{r})}$ is nonempty and the last right vertex of $\Delta_{h}$ lies on the vertical axis. Let $L$ be the last segment of $\mathcal{N}_{h}^{(\mathbf{r})}$.

$\left({ }^{1}\right) F_{2}$ is the projection of $F$ on the vertical axis. 
Then the polygon $\mathcal{N}_{\partial h / \partial X}^{(\mathbf{r})}$ has at least one upper nonstandard segment if and only if there exists a point of $\operatorname{supp} h$ with positive abscissa in the interior of the strip $\mathbb{R} \times L_{2}$. Moreover:

(a) For every upper nonstandard segment $R \in \mathcal{N}_{\partial h / \partial X}^{(\mathbf{r})}$ we have

$$
\frac{\left|L_{1}\right|}{\left|L_{2}\right|} \sigma(L) \leq \frac{\left|R_{1}\right|}{\left|R_{2}\right|} \sigma(R) .
$$

(b) If $R \in \mathcal{N}_{\partial h / \partial X}^{(\mathbf{r})}$ is an upper nonstandard segment parallel to $L$, then

$$
\operatorname{in}\left(\frac{\partial h}{\partial X}, R\right)=\frac{\partial}{\partial X} \operatorname{in}(h, L) \text {. }
$$

ExAmple. Let $h=Y+X Y^{2}+X^{4} Y^{3}+Y^{9}+X^{3} Y^{7}+X^{8} Y^{5}+X^{8} Y^{7}$.
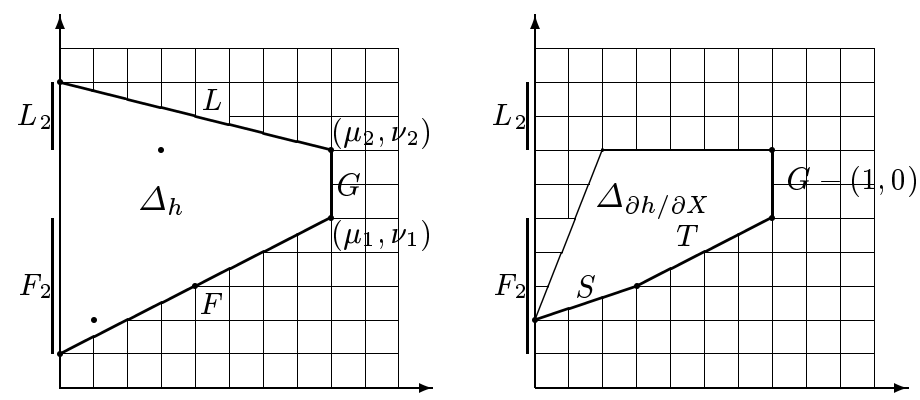

The right polygon of $\mathcal{N}_{h}$ consists of three segments $F, G$ and $L$ which join $(0,1),(8,5)$ and $(0,9)$. The right polygon of $\partial h / \partial X=Y^{2}+4 X^{3} Y^{3}+$ $3 X^{2} Y^{7}+8 X^{7} Y^{5}+8 X^{7} Y^{7}$ consists of two lower nonstandard segments $S, T$ and one standard segment $G-(1,0)$. The reason for the lack of upper nonstandard segments is the lack of points from $\operatorname{supp} h$ with positive ordinates in the interior of $\mathbb{R} \times L_{2}$.

A straightforward consequence of Propositions 8.3 and 8.4 is

Corollary 8.5. Let $h \in \mathbb{C}[X, Y]$ and let $R \in \mathcal{N}_{\partial h / \partial X}^{(\mathbf{r})}$ (notice that $\mathcal{N}_{h}^{(\mathbf{r})}$ is nonempty). Then:

(a) If $R$ is parallel to a segment $S \in \mathcal{N}_{h}^{(\mathbf{r})}$, then

$$
\operatorname{in}\left(\frac{\partial h}{\partial X}, R\right)=\frac{\partial}{\partial X} \operatorname{in}(h, S) \text {. }
$$

(b) If $R$ is not parallel to any segment of $\mathcal{N}_{h}^{(\mathbf{r})}$, then exactly one of the following possibilities holds: 
(i) The declivity of $R$ is less than all the declivities of the segments from $\mathcal{N}_{h}^{(\mathbf{r})}$; such an $R$ can only exist if the first right vertex of $\Delta_{h}$ lies on the vertical axis.

(ii) The declivity of $R$ is greater than all the declivities of the segments from $\mathcal{N}_{h}^{(\mathbf{r})}$; such an $R$ can only exist if the last right vertex of $\Delta_{h}$ lies on the vertical axis.

A consequence of the above considerations is the following theorem on nondegeneracy inheritance.

TheOREm 8.6. Let $h \in \mathbb{C}[X, Y]$ be a polynomial without constant term, which is nondegenerate on each segment of its Newton polygon at infinity. Then the pair of its derivatives is also nondegenerate at infinity.

P r o of. Let $R$ be an arbitrary segment of the polygon of $\partial h / \partial X$ at infinity, and let $T$ be an arbitrary segment of the polygon of $\partial h / \partial Y$ at infinity. We have to show that at least one of the conditions from the definition of pair-nondegeneracy is satisfied (Section 3).

Without loss of generality, we can assume that $R$ and $T$ are parallel, and both belong to the right polygons. The existence of these segments implies that $\mathcal{N}_{h}^{(\mathbf{r})}$ is nonempty. We claim that there exists $S \in \mathcal{N}_{h}^{(\mathbf{r})}$ parallel to $R$ and $T$. If not, then by Corollary 8.2 the declivity of $T$ is less than the declivities of all segments from $\mathcal{N}_{h}^{(\mathbf{r})}$. Moreover, the first right vertex of $\Delta_{h}$ lies on the horizontal axis. Since $h$ is without a constant term, this vertex does not lie on the vertical axis. This means, by Corollary 8.5, that the declivity of $R$ is greater than the declivities of all segments from $\mathcal{N}_{h}^{(\mathbf{r})}$, contrary to $R$ and $T$ being parallel. So, such a segment $S$ has to exist. From Corollaries 8.2 and 8.5 it follows that

$$
\operatorname{in}\left(\frac{\partial h}{\partial X}, R\right)=\frac{\partial}{\partial X} \operatorname{in}(h, S) \text { and } \operatorname{in}\left(\frac{\partial h}{\partial Y}, T\right)=\frac{\partial}{\partial Y} \operatorname{in}(h, S) .
$$

That means that the system in $(\partial h / \partial X, R)=\operatorname{in}(\partial h / \partial Y, T)=0$ has no solution in $(\mathbb{C} \backslash\{0\}) \times(\mathbb{C} \backslash\{0\})$ since the system $\frac{\partial}{\partial X} \operatorname{in}(h, S)=\frac{\partial}{\partial Y} \operatorname{in}(h, S)=0$ has none. This ends the proof of the theorem.

9. Proof of the main result. In this section we end the proof of the main result (Theorem 2.1). Considering Theorems 3.1, 3.2 and 8.6, all we have to do is to show that the minimum of the six quantities

$$
\begin{aligned}
& \text { deg } \operatorname{grad} h(X, 0) \text {, } \\
& \inf _{R \in \mathcal{N}_{\partial h / \partial X}^{(\mathbf{r})}} \alpha\left(R, \Delta_{\partial h / \partial Y}\right), \quad \inf _{T \in \mathcal{N}_{\partial h / \partial Y}^{(\mathbf{r})}} \alpha\left(T, \Delta_{\partial h / \partial X}\right), \\
& \text { (21) deg } \operatorname{grad} h(0, Y) \text {, } \\
& \inf _{R \in \mathcal{N}_{\partial h / \partial X}^{(\mathbf{t})}} \beta\left(R, \Delta_{\partial h / \partial Y}\right), \quad \inf _{T \in \mathcal{N}_{\partial h / \partial Y}^{(\mathbf{t})}} \beta\left(T, \Delta_{\partial h / \partial X}\right),
\end{aligned}
$$


is equal to the minimum from the statement of Theorem 2.1. Let us start with a simple

Proposition 9.1. Let $h \in \mathbb{C}[X, Y]$ be a nonzero polynomial not divisible by $Y^{2}$. Then the right polygon of $h$ has a segment which is not exceptional if and only if $\operatorname{deg}_{Y} h \geq 2$.

Proof. Let $S$ be a segment of the right polygon of $h$ which is not exceptional. The ordinate of the upper end of $S$ is greater than 1 . So, $\operatorname{deg}_{Y} h \geq 2$. To prove the opposite implication, consider the right vertex of $\Delta_{h}$ with maximal ordinate. Since $\operatorname{deg}_{Y} h \geq 2$, this ordinate is also $\geq 2$. The vertex is the upper end of a segment, because from the assumption that $h$ is not divisible by $Y^{2}$ it follows that there exists a point in $\operatorname{supp} h$ with ordinate 0 or 1 . Obviously, this segment is not exceptional.

By symmetry we obtain

Proposition 9.2. Let $h \in \mathbb{C}[X, Y]$ be a nonzero polynomial not divisible by $X^{2}$. Then the top polygon of $h$ has a segment which is not exceptional if and only if $\operatorname{deg}_{X} h \geq 2$.

The desired equality of minima can be derived from the following two lemmas:

Lemma 9.3. Let $h \in \mathbb{C}[X, Y]$ be a polynomial without constant term such that $\partial h / \partial X$ is nonzero and $h$ is not divisible by $Y^{2}$. Suppose that the right polygon of $h$ contains at least one segment which is not exceptional, and denote by $F$ the first such segment. Let $m^{(\mathbf{r})}$ be the minimum of the three numbers from (20). Then

$$
\alpha(F)-1 \geq m^{(\mathbf{r})} \geq \min \left\{\alpha(F)-1, \alpha(F)-\frac{\left|F_{1}\right|}{\left|F_{2}\right|} \sigma(F)\right\} .
$$

Lemma 9.4. Let $h \in \mathbb{C}[X, Y]$ be a polynomial without constant term such that $\partial h / \partial Y$ is nonzero and $h$ is not divisible by $X^{2}$. Suppose that the polygon $\mathcal{N}_{h}^{(\mathbf{t})}$ contains at least one segment which is not exceptional, and denote by $G$ the first such segment. Let $m^{(\mathbf{t})}$ be the minimum of the three numbers from (21). Then

$$
\beta(G)-1 \geq m^{(\mathbf{t})} \geq \min \left\{\beta(G)-1, \beta(G)-\frac{\left|G_{2}\right|}{\left|G_{1}\right|} \sigma(G)\right\} .
$$

Obviously, Lemma 9.4 can be obtained from the previous one by symmetry. Before the proof of Lemma 9.3 let us see how the lemmas imply the desired equality.

Suppose that $h \in \mathbb{C}[X, Y]$ satisfies the assumptions of Theorem 2.1. In particular, at least one of the polygons $\mathcal{N}_{h}^{(\mathbf{r})}, \mathcal{N}_{h}^{(\mathbf{t})}$, say $\mathcal{N}_{h}^{(\mathbf{r})}$, has a segment 
which is not exceptional. Denote by $F$ the first such segment. We consider two possibilities: $\operatorname{deg}_{X} h \leq 1$ or $\operatorname{deg}_{X} h \geq 2$.

If $\operatorname{deg}_{X} h \leq 1$, then, by Proposition 9.2, the top polygon of $h$ is empty or has only the exceptional segment. So, it is sufficient to show that the minimum of the six quantities from (20) and $(21)$ is $\alpha(F)-1$. We have $\left|S_{2}\right| \geq 1$ for any segment $S$ in the right polygon. So, $\left|F_{2}\right| \geq 1$. From the assumption $\operatorname{deg}_{X} h \leq 1$ it follows that $\left|F_{1}\right| \leq 1$. Hence, by Lemma 9.3, the minimum of the three quantities in $(20)$ is $\alpha(F)-1$. Notice that $h=$ $a(Y) X+b(Y)$, and since $\partial h / \partial X \neq 0$, we have $a \neq 0$. One can easily verify that deg $\operatorname{grad} h(X, 0) \leq 1$, which gives $\alpha(F)-1 \leq 1$.

To end the proof in this case, it is enough to show that each of the three quantities in (21) is $\geq 1$. By Proposition 9.1 we have $\operatorname{deg}_{Y} h=$ $\max \{\operatorname{deg} a, \operatorname{deg} b\} \geq 2$, hence $\operatorname{deg} \operatorname{grad} h(0, Y) \geq 1$. Since the top polygon of $\partial h / \partial X$ is empty, the second quantity is $+\infty$. The third quantity is also $+\infty$ if the top polygon of $\partial h / \partial Y$ is empty. If it is nonempty, then obviously $\operatorname{deg} a \geq 1$. Since $\operatorname{deg} \frac{\partial h}{\partial X}(0, Y)=\operatorname{deg} a$, we have $(0, \operatorname{deg} a) \in \operatorname{supp} \partial h / \partial X$. By definition (3) we obtain $\beta\left(T, \Delta_{\partial h / \partial X}\right) \geq \operatorname{deg} a \geq 1$ for any $T$ such that $\left|T_{1}\right| \neq 0$. This ends the proof in the case considered.

Now, assume that $\operatorname{deg}_{X} h \geq 2$. By Proposition 9.2 there exists a segment of $\mathcal{N}_{h}^{(\mathbf{t})}$ which is not exceptional. Let $G$ be the first such segment. Denote by $m_{1}$ the minimum of

$$
\alpha(F)-1, \quad \beta(G)-1,
$$

and by $m_{2}$ the minimum of

$$
\alpha(F)-\frac{\left|F_{1}\right|}{\left|F_{2}\right|} \sigma(F), \quad \beta(G)-\frac{\left|G_{2}\right|}{\left|G_{1}\right|} \sigma(G) .
$$

By Lemmas 9.1 and 9.2 the minimum of the six quantities in (20) and (21) is at most $m_{1}$ and at least $\min \left\{m_{1}, m_{2}\right\}$. To end the proof it suffices to show that $m_{1} \leq m_{2}$. All is clear if $\frac{\left|F_{1}\right|}{\left|F_{2}\right|} \sigma(F) \leq 1$ and $\frac{\left|G_{2}\right|}{\left|G_{1}\right|} \sigma(G) \leq 1$. So, suppose that $\frac{\left|F_{1}\right|}{\left|F_{2}\right|} \sigma(F)>1$. In particular, $\sigma(F)=1$ and $\left|F_{1}\right|>\left|F_{2}\right|$. It is enough to show that $\beta(G)-1 \leq m_{2}$. Notice that the segment $F$ belongs to the top polygon $\mathcal{N}_{h}^{(\mathbf{t})}$ and its declivity in this polygon, $\frac{\left|F_{2}\right|}{\left|F_{1}\right|} \sigma(F)$, is less than 1 . Since $\left|F_{1}\right|>\left|F_{2}\right| \geq 1, F$ cannot be exceptional in $\mathcal{N}_{h}^{(\mathbf{t})}$. Hence the declivity of $G$ in the top polygon is no more than that of $F$. So,

$$
\frac{\left|G_{2}\right|}{\left|G_{1}\right|} \sigma(G) \leq \frac{\left|F_{2}\right|}{\left|F_{1}\right|} \sigma(F)<1,
$$

and therefore

$$
\beta(G)-1 \leq \beta(G)-\frac{\left|G_{2}\right|}{\left|G_{1}\right|} \sigma(G) .
$$


Moreover, $\beta(G) \leq \beta(F)$ and $\beta(F)-1 \geq 0$. Hence

$$
\beta(G)-1 \leq[\beta(F)-1] \frac{\left|F_{1}\right|}{\left|F_{2}\right|} \sigma(F)=\alpha(F)-\frac{\left|F_{1}\right|}{\left|F_{2}\right|} \sigma(F) .
$$

Above we use the simple equality $\beta(S) \frac{\left|S_{1}\right|}{\left|S_{2}\right|} \sigma(S)=\alpha(S)$ (with $S=F$ ), which holds for every $S$ such that $\left|S_{2}\right| \neq 0$. This ends the proof of $m_{1} \leq m_{2}$ in the situation considered. The case $\frac{\left|G_{2}\right|}{\left|G_{1}\right|} \sigma(G)>1$ can be verified analogously.

Now we give the proof of the lemma.

Proof of Lemma 9.3. First, we show that for any segment $S$ of $\mathcal{N}_{h}^{(\mathbf{r})}$,

$$
\alpha\left(S, \Delta_{\partial h / \partial X}\right)=\alpha(S)-1 \quad \text { and } \quad \alpha\left(S, \Delta_{\partial h / \partial Y}\right)=\alpha(S)-\frac{\left|S_{1}\right|}{\left|S_{2}\right|} \sigma(S) .
$$

To prove the first equality, consider the linear form

$$
\phi(\alpha, \beta)=\alpha+\beta \frac{\left|S_{1}\right|}{\left|S_{2}\right|} \sigma(S) .
$$

By the definition (2) and by the inclusion supp $\partial h / \partial X \subset \operatorname{supp} h-(1,0)$ we have

$$
\begin{aligned}
\alpha\left(S, \Delta_{\partial h / \partial X}\right) & =\inf \phi\left(\operatorname{supp} \frac{\partial h}{\partial X}\right) \geq \inf \phi(\operatorname{supp} h)+\phi(-1,0) \\
& =\alpha\left(S, \Delta_{h}\right)-1 .
\end{aligned}
$$

Using the elementary fact $\alpha(S)=\alpha\left(S, \Delta_{h}\right)$ for $S \in \mathcal{N}_{h}^{(\mathbf{r})}$, we obtain the inequality $\geq$. To prove the opposite inequality, notice that from $\partial h / \partial X \neq 0$ it follows that at least one of the ends of $S$ has a positive abscissa. Denote it by $(\alpha, \beta)$. Since $(\alpha-1, \beta) \in \operatorname{supp} \partial h / \partial X$, we have

$$
\alpha\left(S, \Delta_{\partial h / \partial X}\right) \leq(\alpha-1)+\beta \frac{\left|S_{1}\right|}{\left|S_{2}\right|} \sigma(S) .
$$

This ends the proof since $\alpha(S)=\alpha+\beta \frac{\left|S_{1}\right|}{\left|S_{2}\right|} \sigma(S)$ for any $(\alpha, \beta) \in S$. The second equality in (22) can be proved analogously.

Now, we prove the inequalities of Lemma 9.3. Let $(\mu, \nu)$ be the first right vertex of the diagram $\Delta_{h}$ with positive ordinate. We consider two cases: $\nu=1$ and $\nu \geq 2$. In both, we show that each quantity from (20) is no more than $\alpha(F)-1$, or $\alpha(F)-\frac{\left|F_{1}\right|}{\left|F_{2}\right|} \sigma(F)$. Moreover, we show that at least one of the quantities does not exceed $\alpha(F)-1$.

Assume that $\nu=1$. Since $F$ is the first segment of $\mathcal{N}_{h}^{(\mathbf{r})}$ which is not exceptional, the vertex $(\mu, \nu)=(\mu, 1)$ is the lower end of $F$. Since $(\mu, 1) \in F$, we have $\alpha(F)=\mu+\frac{\left|F_{1}\right|}{\left|F_{2}\right|} \sigma(F)$. Notice that $(\mu, 0)$ is the vertex of $\Delta_{\partial h / \partial Y}$, therefore $\operatorname{deg} \frac{\partial h}{\partial Y}(X, 0)=\mu$. Hence

$$
\operatorname{deg} \operatorname{grad} h(X, 0) \geq \operatorname{deg} \frac{\partial h}{\partial Y}(X, 0)=\mu=\alpha(F)-\frac{\left|F_{1}\right|}{\left|F_{2}\right|} \sigma(F) .
$$


Note the general fact that for any nonzero $f \in \mathbb{C}[X, Y]$ and any $S$ such that $\left|S_{2}\right| \neq 0$ we have $\alpha\left(S, \Delta_{f}\right) \geq \operatorname{deg} f(X, 0)$. For $f(X, 0)=0$ this is obvious. If $f(X, 0) \neq 0$, then it follows from (2) and from the fact that $(\operatorname{deg} f(X, 0), 0) \in \operatorname{supp} f$. Applying this fact to $\partial h / \partial Y$ we obtain

$$
\inf _{R \in \mathcal{N}_{\partial h / \partial X}^{(\mathbf{r})}} \alpha\left(R, \Delta_{\partial h / \partial Y}\right) \geq \mu=\alpha(F)-\frac{\left|F_{1}\right|}{\left|F_{2}\right|} \sigma(F) .
$$

To estimate the third quantity in $(20)$, notice that $F-(0,1)$ is the first segment of the right polygon of $\partial h / \partial Y$. That means that it has the minimal declivity in this polygon. The value $\alpha\left(\cdot, \Delta_{\partial h / \partial X}\right)$ does not change if we replace $F-(0,1)$ by the parallel $F$. Hence, by the second equality in $(22)$ we obtain

$$
\inf _{T \in \mathcal{N}_{\partial h / \partial Y}^{(\mathbf{r})}} \alpha\left(T, \Delta_{\partial h / \partial X}\right)=\alpha\left(F, \Delta_{\partial h / \partial X}\right)=\alpha(F)-1 .
$$

Combining the above equality with estimates (23) and (24) we end the proof of the lemma in this case.

Now, assume that $\nu \geq 2$. We claim that the first right vertex of $\Delta_{h}$ lies on the horizontal axis. If not, then this vertex coincides with $(\mu, \nu)$, and by $\nu \geq 2, h$ is divisible by $Y^{2}$, a contradiction. So, let $(p, 0)$ be the first vertex of $\Delta_{h}$. Obviously, $p=\alpha(F)$. Since $h$ has no constant term, we have $p \geq 1$. Hence, $(p-1,0)$ is a right vertex of $\Delta_{\partial h / \partial X}$ and $\operatorname{deg} \frac{\partial h}{\partial X}(X, 0)=p-1=$ $\alpha(F)-1$. As before we have

and

$$
\operatorname{deg} \operatorname{grad} h(X, 0) \geq \operatorname{deg} \frac{\partial h}{\partial X}(X, 0)=\alpha(F)-1
$$

$$
\inf _{T \in \mathcal{N}_{\partial h / \partial Y}^{(\mathbf{r})}} \alpha\left(T, \Delta_{\partial h / \partial X}\right) \geq \operatorname{deg} \frac{\partial h}{\partial X}(X, 0)=\alpha(F)-1 .
$$

To estimate the third quantity, notice that the declivities of all segments from the right polygon of $\partial h / \partial X$ are greater than or equal to the declivity of the first segment $F$ of $\mathcal{N}_{h}^{(\mathbf{r})}$. This follows from Corollary 8.5, because the first right vertex $(p, 0)$ of $\Delta_{h}$ does not lie on the vertical axis. Hence

$$
\inf _{R \in \mathcal{N}_{\partial h / \partial X}^{(\mathbf{r})}} \alpha\left(R, \Delta_{\partial h / \partial Y}\right) \geq \alpha\left(F, \Delta_{\partial h / \partial Y}\right)=\alpha(F)-\frac{\left|F_{1}\right|}{\left|F_{2}\right|} \sigma(F) .
$$

To end the proof of the lemma it is sufficient to show that in any case, at least one of the quantities in (20) is less than or equal to $\alpha(F)-1$. We consider two cases. If there exists a nonstandard segment of the right polygon of $\partial h / \partial Y$, then by Proposition 8.1, its declivity does not exceed the declivity of $F$. Hence

$$
\inf _{T \in \mathcal{N}_{\partial h / \partial Y}^{(\mathbf{r})}} \alpha\left(T, \Delta_{\partial h / \partial X}\right) \leq \alpha\left(F, \Delta_{\partial h / \partial X}\right)=\alpha(F)-1 .
$$


In the opposite case, by the same proposition, $\operatorname{supp} h \cap\{0<\beta<\nu\}$ is empty, which, as $\nu \geq 2$, means that $\frac{\partial h}{\partial Y}(X, 0)=0$. Hence,

$$
\text { deg } \operatorname{grad} h(X, 0)=\max \left\{\operatorname{deg} \frac{\partial h}{\partial X}(X, 0),-\infty\right\}=\alpha(F)-1 .
$$

This ends the proof of the lemma and the proof of the main result.

Acknowledgments. This research was partially supported by the State Committee for Scientific Research of Poland, grant No. 2 PO3A 07908. The author would like to thank The Fields Institute and the University of Toronto for hospitality and support during finishing this paper.

\section{References}

[A] M. Artin, On the solution of analytic equations, Invent. Math. 5 (1968), 277291.

[BK] E. Brieskorn und H. Knörrer, Ebene algebraische Kurven, Birkhäuser, 1981.

[CN-H] P. Cassou-Noguès et Há Huy Vui, Sur le nombre de Eojasiewicz à l'infini d'un polynôme, Ann. Polon. Math. 62 (1995), 23-44.

[ChK1] J. Chądzyński and T. Krasiński, Exponent of growth of polynomial mappings of $\mathbb{C}^{2}$ into $\mathbb{C}^{2}$, in: Singularities, Banach Center Publ. 20, PWN, Warszawa, 1988, $147-160$.

[ChK2] - - - On the Eojasiewicz exponent at infinity for polynomial mappings of $\mathbb{C}^{2}$ into $\mathbb{C}^{2}$ and components of polynomial automorphisms of $\mathbb{C}^{2}$, Ann. Polon. Math. 57 (1992), 291-302.

[L] A. Lenarcik, On the Eojasiewicz exponent of the gradient of a holomorphic function, in: Singularities Symposium-Łojasiewicz 70, Banach Center Publ. 44, Inst. Math., Polish Acad. Sci., Warszawa, 1998, 149-166.

[Pł1] A. Płoski, On the growth of proper polynomial mappings, Ann. Polon. Math. 45 (1985), 297-309.

[Pł2] - Newton polygons and the Eojasiewicz exponent of a holomorphic mapping of $\mathbb{C}^{2}$, ibid. 51 (1990), 275-281.

[W] R. Walker, Algebraic Curves, Princeton Univ. Press, 1950.

Kielce University of Technology

Aleja Tysiąclecia Państwa Polskiego 7

25-314 Kielce, Poland

E-mail: lenarcik@sabat.tu.kielce.pl 